\title{
Jackiw-Teitelboim gravity and rotating black holes
}

\author{
Upamanyu Moitra, Sunil Kumar Sake, Sandip P. Trivedi and V. Vishal \\ Department of Theoretical Physics, Tata Institute of Fundamental Research, \\ Mumbai 400005, India \\ E-mail: upamanyu@theory.tifr.res.in, sunil.sake@tifr.res.in, \\ sandip@theory.tifr.res.in, vishal@theory.tifr.res.in
}

ABSTRACT: We show that the free energy at low temperatures for near-extremal black holes is correctly obtained from the Jackiw-Teitelboim (JT) model of gravity. Our arguments apply to all black holes, including rotating ones, whose metric has a near-horizon $\mathrm{AdS}_{2}$ factor and the associated $\mathrm{SL}(2, \mathbb{R})$ symmetry. We verify these arguments by explicit calculations for rotating black holes in 4 and 5 dimensions. Our results suggest that the JT model could prove useful in analysing the dynamics of near-extremal Kerr black holes found in nature.

KEYwords: 2D Gravity, AdS-CFT Correspondence, Black Holes, Models of Quantum Gravity

ArXiv EPrint: 1905.10378 


\section{Contents}

1 Introduction 1

2 A review of the Jackiw-Teitelboim model and near-extremal ReissnerNordström black holes $\quad 3$

2.1 Jackiw-Teitelboim model 3

2.2 Near-extremal Reissner-Nordström black holes 5

3 A more detailed comparison with the JT model 9

$\begin{array}{llr}3.1 & \text { RN black holes } & 9\end{array}$

3.1.1 Some comments 11

$\begin{array}{lll}3.2 & \text { More general theories } & 12\end{array}$

$\begin{array}{lll}3.3 & \text { Rotating black holes } & 14\end{array}$

4 Five-dimensional rotating black holes in asymptotically Anti-de Sitter $\begin{array}{ll}\text { spacetime } & 19\end{array}$

5 Four-dimensional charged rotating black holes $\quad 22$

5.1 The near-extremal Kerr black hole 24

$\begin{array}{ll}5.2 \text { Superradiant instabilities } & 26\end{array}$

6 Conclusions $\quad 26$

A On how the Jackiw-Teitelboim model correctly reproduces the leading $\begin{array}{ll}\text { thermodynamic behaviour } & 29\end{array}$

B Electrically charged near-extremal black holes in $(d+1)$ dimensions $\quad 32$

C Rotating black holes in five dimensions in asymptotically Anti-de Sitter $\begin{array}{ll}\text { spacetime } & 35\end{array}$

$\begin{array}{lll}\text { C.1 Dimensional reduction to two dimensions } & 36\end{array}$

D Rotating dyonic black holes in four dimensions in asymptotically Anti-de $\begin{array}{ll}\text { Sitter spacetime } & 37\end{array}$

D.1 Dimensional reduction to two dimensions 40

\section{Introduction}

Exciting recent developments have revealed that the behaviour of a class of models studied in condensed matter physics, which we will refer to generically as Sachdev-Ye-Kitaev (SYK) models here [1, 2], is connected with the behaviour of near-extremal black holes. 
More precisely, it was shown in [3] that the behaviour of the Jackiw-Teitelboim (JT) theory [4-6] of 2-dimensional gravity has many parallels with the SYK model. In particular, in the SYK model, conformal invariance is broken, and this breaking determines the low temperature thermodynamics and low-energy response of the system. Similarly, conformal invariance is also broken in the JT model, and this breaking again determines the thermodynamics and response of the system.

In subsequent works, $[7,8]$ it was shown that in fact the JT model is a good approximation for the dynamics and thermodynamics of a large class of charged black holes close to extremality. And by understanding the breaking of conformal invariance, a precise version of the near- $\mathrm{AdS}_{2} /$ near-CFT $\mathrm{C}_{1}$ correspondence was also developed which applies to these black holes. The only requirement for these considerations to apply is that the nearhorizon geometry at extremality has an $\mathrm{AdS}_{2}$ factor and the $\mathrm{SL}(2, \mathbb{R})$ symmetry associated with it. This is true for the Reissner-Nordström black hole, and several other black holes which can arise in string theory, including those in systems which have extra scalars and gauge fields.

In this paper, we extend these considerations to rotating black holes. Several examples of extremal rotating black holes including the extremal Kerr black hole in flat or asymptotically AdS spacetime are known to posses an $\operatorname{AdS}_{2}$ factor and an $\operatorname{SL}(2, \mathbb{R})$ symmetry in their near-horizon geometry. We show that the low-temperature thermodynamics, close to extremality, for these black holes is correctly obtained from a JT model with appropriate values for the two-dimensional Newton's constant and the scale of conformal symmetry breaking. This is the central result of the paper.

The paper is structured as follows. After a review of the connection between the JT model and near-extremal Reissner-Nordström black holes in section 2, we give an explanation for why the JT model should give the correct low temperature free energy and associated thermodynamics for all spherically symmetric near-extremal black holes with a near-horizon $\mathrm{AdS}_{2}$ geometry in section 3.1. These arguments are extended to the rotating case in general in section 3.3, once again for all cases where the extremal geometry has an $\mathrm{AdS}_{2}$ near-horizon geometry.

In the following two sections, 4 and 5, we consider several examples, including the near-extremal Kerr black hole in 5 dimensions in asymptotically AdS spacetime, the nearextremal dyonic Kerr-Newman black hole in asymptotically AdS spacetime in 4 dimensions, and in particular, the near-extremal Kerr black hole in 4 dimensions in asymptotically flat spacetime. By explicit calculations, we show that the general conclusion referred to above is correct, namely the low temperature behaviour of their free energy agrees with that of the JT model.

Our results about the wide applicability of the JT model for the rotating case as well, suggests, in fact, that this model could be useful for studying some aspects of the dynamics of near-extremal Kerr black holes found in nature. We end the paper by making some comments along these lines, along with a summary and some conclusions in section 6 .

Appendices A-D contain some important additional supplementary material.

Before we proceed, let us mention some of the relevant literature. The various aspects of the SYK model, its variants and the JT model have also been studied in, e.g., [9-117]. For 
a pedagogical review of the SYK model, see [118]; the connections with $\mathrm{AdS}_{2}$ holography are reviewed in [119]. The Kerr-CFT correspondence was studied earlier in [120] and was further explored in, for instance, [121-129]; for a review, see [130].

\section{A review of the Jackiw-Teitelboim model and near-extremal Reissner- Nordström black holes}

\section{$2.1 \quad$ Jackiw-Teitelboim model}

Let us briefly recapitulate some of the key features of the Jackiw-Teitelboim (JT) model of two-dimensional gravity and how it describes near-extremal Reissner-Nordström (RN) black holes. The JT model consists of two-dimensional gravity with a dilaton $\phi$ described by the action:

$$
I=-\frac{1}{16 \pi \widetilde{G}}\left(\int \mathrm{d}^{2} x \sqrt{g} \phi\left(R-\Lambda_{2}\right)+2 \int_{\partial} \mathrm{d} x \sqrt{\gamma} \phi K\right)
$$

Note that we are working in Euclidean spacetime here since this paper is mostly concerned with black hole thermodynamics.

The two-dimensional spacetimes one considers in the model have a one-dimensional boundary, denoted by $\partial$ in eq. (2.1), at a fixed value of the dilaton

$$
\phi=\phi_{B}
$$

In addition, fluctuations in the metric also vanish sufficiently fast at the boundary; we will be more precise about this shortly.

The equations of motion then lead to the geometry being $\mathrm{AdS}_{2}$

$$
\mathrm{d} s^{2}=\frac{L_{2}^{2}}{z^{2}}\left(\mathrm{~d} t^{2}+\mathrm{d} z^{2}\right)
$$

with radius $L_{2}$ being given by,

$$
L_{2}=\sqrt{-\frac{2}{\Lambda_{2}}}
$$

In addition, the dilaton can be linearly varying

$$
\phi=\frac{1}{\mathcal{J} z} .
$$

This linear variation breaks the scale invariance of $\mathrm{AdS}_{2}$ and is parametrised by the scale $\mathcal{J}$ which has dimensions of $[M] \sim[L]^{-1}$. This breaking of scale invariance is very important in the resulting behaviour of the system.

The boundary conditions imposed on the metric can be now stated more precisely. In a coordinate system with $g_{t z}=0$,

$$
\begin{aligned}
g_{t t} & \rightarrow \frac{L_{2}^{2}}{z^{2}}\left(1+\mathcal{O}\left(z^{2}\right)\right), \\
g_{z z} & \rightarrow \frac{L_{2}^{2}}{z^{2}}\left(1+\mathcal{O}\left(z^{2}\right)\right),
\end{aligned}
$$


as $z \rightarrow z_{B}$, where $\phi=\phi_{B}$. As was discussed in [7], these boundary conditions, along with the Dirichlet boundary condition for the dilaton, eq. (2.2), give rise to a well-defined variational principle, with the equations of motion having a solution, eqs. (2.3), (2.5).

In our discussion below, where the JT model arises from black holes in higher dimensions, $z_{B}$ will be small enough at the boundary so that the $\mathcal{O}\left(z^{2}\right)$ and higher corrections in eq. (2.6) are suppressed, but not too small, so that, eq. (2.5),

$$
\phi_{B}=\frac{1}{\mathcal{J} z_{B}}
$$

is also small meeting the condition, $\phi_{B} \ll 1$.

The dynamics of the system arise from fluctuations of the boundary which can be parametrised by time reparametrisations and is described by a Schwarzian action,

$$
I=-\frac{1}{8 \pi \widetilde{G} \mathcal{J}} \int \mathrm{d} \tau \operatorname{Sch}(t, \tau)
$$

where,

$$
\operatorname{Sch}(t, \tau) \equiv \frac{t^{\prime \prime \prime}(\tau)}{t^{\prime}(\tau)}-\frac{3}{2}\left(\frac{t^{\prime \prime}(\tau)}{t^{\prime}(\tau)}\right)^{2}
$$

Here $t$ is the Poincaré time coordinate in eq. (2.3) and $\tau$ is the proper time along the boundary defined by,

$$
\frac{\mathrm{d} \tau^{2}}{z_{B}^{2}}=\left.\frac{1}{z^{2}}\left(\mathrm{~d} t^{2}+\mathrm{d} z^{2}\right)\right|_{\partial}
$$

where $z_{B}$ is location of the boundary, eq. (2.2) in the $z$ coordinate.

The reader will notice that in the action, eq. (2.1), we have included a Gibbons-Hawking (GH) boundary term,

$$
I_{\mathrm{GH}}=-\frac{1}{8 \pi \widetilde{G}} \int_{\partial} \mathrm{d} x \sqrt{\gamma} \phi K .
$$

In addition to the action eq. (2.1), we also need to add a counter-term,

$$
I_{\mathrm{CT}}=\frac{1}{8 \pi \widetilde{G} L_{2}} \int_{\partial} \mathrm{d} x \sqrt{\gamma} \phi,
$$

which cancels the divergence which arises from the GH term in evaluating the on-shell action, to obtain a finite mass and a finite on-shell action. The ADM mass is then given by

$$
M_{\mathrm{ADM}}=\frac{1}{8 \pi \widetilde{G} \mathcal{J}} \operatorname{Sch}(t, \tau),
$$

and is also proportional to the Schwarzian. The free energy at temperature $T$ of the system is given by

$$
F=-\frac{\pi}{4 \widetilde{G} \mathcal{J}} T^{2},
$$

and is quadratic in $T$. Notice that the scale $\mathcal{J}$ appears in the denominator in the Schwarzian action, mass and free energy. 


\section{$2.2 \quad$ Near-extremal Reissner-Nordström black holes}

Next consider a near-extremal RN geometry in 4 dimensions in either asymptotically flat or $\mathrm{AdS}_{4}$ spacetime with metric

$$
\mathrm{d} s^{2}=f(r) \mathrm{d} t^{2}+\frac{\mathrm{d} r^{2}}{f(r)}+\Phi^{2}(r) \mathrm{d} \Omega_{2}^{2} .
$$

Here,

$$
f(r)=\left(1-\frac{2 G_{4} M}{r}+\frac{Q^{2}}{r^{2}}+\frac{r^{2}}{L^{2}}\right)
$$

and

$$
\Phi(r)=r,
$$

$L$ is the radius of $\mathrm{AdS}_{4}$ and the flat spacetime limit is obtained by taking $L \rightarrow \infty$. For an electrically charged black hole, the electromagnetic field strength is

$$
F_{r t}=\frac{Q}{r^{2}} .
$$

In the extremal limit, $f(r)$ has a "double zero" at a radial location we denote by $r_{h}$ and is given by

$$
f_{0}(r)=\frac{\left(r-r_{h}\right)^{2}}{L_{2}^{2}}
$$

in the vicinity of the horizon. The radial location of the horizon, $r_{h}$, is an important parameter in the subsequent discussion. It determines the radius of the horizon, eq. (2.17) and is fixed by $Q$ and in the asymptotically AdS case, also by the radius of $\operatorname{AdS}_{4}, L$. The near-horizon geometry for an extremal $\mathrm{RN}$ black hole is $\mathrm{AdS}_{2} \times S^{2}$ with the radius $L_{2}$, eq. (2.3), also being determined in general by the charge $Q$, and $L$. The coordinate $z$ in eq. (2.3) is related to the radial coordinate in eq. (2.15) by

$$
z=\frac{L_{2}^{2}}{\left(r-r_{h}\right)} \text {. }
$$

The spherically symmetric geometry in eq. (2.15) is suggestive and we can carry out the dimensional reduction over the $S^{2}$ and construct a 2-dimensional system consisting of gravity and a scalar $\Phi$, which is the radius of the two-sphere. This two-dimensional theory turns out to be well approximated by the JT model which accurately describes the thermodynamics at low temperatures [7]. We review how this connection with the JT model arises next. Before proceeding, let us note that for the electrically charged case we discuss below an additional phase mode is also present. Its action can also be expressed in terms of a term on the boundary,

$$
I_{\text {phase }}=-\mathcal{I} \int_{\partial} \mathrm{d} \tau(\dot{\theta})^{2} .
$$

How this mode arises and how the resulting value of the coefficient, $\mathcal{I}$, which is related to the charge susceptibility [31], is obtained was discussed in some detail in [8]. We mention 
it only in passing here since we will mainly study the free energy at fixed charge in this paper and the phase mode is not very relevant in the resulting analysis.

We start with the 4-dimensional theory described by the Euclidean action,

$$
I=-\frac{1}{16 \pi G_{4}}\left(\int \mathrm{d}^{4} x \sqrt{g}\left(R-2 \Lambda_{4}+F_{\mu \nu} F^{\mu \nu}\right)+2 \int \mathrm{d}^{3} x \sqrt{\gamma} K\right) .
$$

Taking the metric of the form

$$
\mathrm{d} s^{2}=g_{\alpha \beta} \mathrm{d} x^{\alpha} \mathrm{d} x^{\beta}+\Phi^{2} \mathrm{~d} \Omega_{2}^{2},
$$

and dimensionally reducing over the $S^{2}$ (here the field $\Phi$ and the metric depend only on the 2 coordinates $x^{\alpha}$ ) and further changing frames under the transformation

$$
g_{\alpha \beta} \rightarrow \frac{\Phi_{0}}{\Phi} g_{\alpha \beta}
$$

gives the two-dimensional action,

$$
I=-\frac{1}{4 G_{4}} \int \mathrm{d}^{2} x \sqrt{g}\left(\Phi^{2} R+\frac{2 \Phi_{0}}{\Phi}-2 \Phi_{0} \Phi \Lambda_{4}+\frac{\Phi^{3}}{\Phi_{0}} F^{\alpha \beta} F_{\alpha \beta}\right)-\frac{1}{2 G_{4}} \int \mathrm{d} x \sqrt{\gamma} \Phi^{2} K .
$$

This action has a solution where the metric is $\mathrm{AdS}_{2}, \Phi$ takes the attractor value

$$
\Phi_{0}=r_{h}=\frac{L}{\sqrt{6}}\left(\sqrt{1+\frac{12 Q^{2}}{L^{2}}}-1\right)^{1 / 2},
$$

and the field strength is given by

$$
F_{\alpha \beta} \mathrm{d} x^{\alpha} \wedge \mathrm{d} x^{\beta}=\frac{Q \Phi_{0}}{\Phi^{3}} \sqrt{g} \epsilon_{\alpha \beta} \mathrm{d} x^{\alpha} \wedge \mathrm{d} x^{\beta},
$$

where $\epsilon_{\alpha \beta}$ is the Levi-Civita symbol (anti-symmetric tensor density). Note that throughout this paper, we have chosen the convention that in the $r, t$ coordinates, $\epsilon_{r t}=1$.

The on-shell value of the action $I$ is known to give the grand canonical partition function. As was mentioned above, here we will consider the canonical partition function. This is obtained by adding a boundary term to $I[131]$,

$$
I_{\partial \mathrm{GF}}=\frac{1}{G_{4}} \int \mathrm{d} x \sqrt{\gamma} \frac{\Phi^{3}}{\Phi_{0}} n_{\alpha} F^{\alpha \beta} A_{\beta} .
$$

in the resulting two-dimensional theory, eq. (2.25). Here $n_{\alpha}$ is the outward normal one-form on the boundary. After including it, we get,

$$
\begin{aligned}
I_{\text {canonical }}= & -\frac{1}{4 G_{4}} \int \mathrm{d}^{2} x \sqrt{g}\left(\Phi^{2} R+\frac{2 \Phi_{0}}{\Phi}-2 \Phi_{0} \Phi \Lambda_{4}+\frac{\Phi^{3}}{\Phi_{0}} F^{\alpha \beta} F_{\alpha \beta}\right) \\
& -\frac{1}{2 G_{4}} \int \mathrm{d} x \sqrt{\gamma}\left(\Phi^{2} K-\frac{2 \Phi^{3}}{\Phi_{0}} n_{\alpha} F^{\alpha \beta} A_{\beta}\right) .
\end{aligned}
$$

Expanding about the attractor value for $\Phi$, eq. (2.26),

$$
\Phi=\Phi_{0}(1+\phi)
$$


and inserting in eq. (2.29) then gives

$$
\begin{aligned}
I= & -\frac{r_{h}^{2}}{4 G_{4}}\left(\int \mathrm{d}^{2} x \sqrt{g} R+2 \int \mathrm{d} x \sqrt{\gamma} K\right) \\
& -\frac{r_{h}^{2}}{2 G_{4}}\left(\int \mathrm{d}^{2} x \sqrt{g} \phi\left(R+\frac{2}{L_{2}^{2}}\right)+2 \int \mathrm{d} x \sqrt{\gamma} \phi K\right) \\
& -\frac{r_{h}^{2}}{4 G_{4}}\left(\int \mathrm{d}^{2} x \sqrt{g} \phi^{2}\left(R-\frac{6}{L_{2}^{2}}-\frac{4}{r_{h}^{2}}\right)+2 \int \mathrm{d} x \sqrt{\gamma} \phi^{2} K\right)+\cdots
\end{aligned}
$$

On the r.h.s. above, the first line, which is independent of $\phi$, gives the entropy for the extremal black hole. For computing the free energy above extremality,

$$
\Delta F=F-F_{\text {extremal }}
$$

as a function of temperature, we can neglect terms in this line. The last line in eq. (2.31) has corrections which are quadratic in $\phi$, as well as additional terms indicated by the ellipsis which include terms with even higher orders in $\phi$. We will also see in the next section why these terms can be neglected for the leading order temperature dependence. This leaves the second line in eq. (2.31) which is exactly the action for the JT model. Comparing with eq. (2.1) we also see that the resulting value of the two-dimensional Newton's constant is,

$$
\widetilde{G}=\frac{G_{4}}{8 \pi r_{h}^{2}} .
$$

In addition to the terms in eq. (2.31), we note that the JT model also has the counter-term, eq. (2.12). We also note that the additional boundary term we added, eq. (2.28), when expanded at linear order in $\phi$ becomes,

$$
I_{\partial \mathrm{GF}}=\frac{r_{h}^{2}}{G_{4}} \int \mathrm{d} x \sqrt{\gamma}(1+3 \phi) n_{\alpha} F^{\alpha \beta} A_{\beta} .
$$

We note that if we treated (2.31) as a two-dimensional action in its own right, including the terms of higher order in $\phi$, the remarkable simplification accorded by the JT model would be lost. However, one can still make progress in principle, using, for example, a perturbative approach - in which the corrections can be kept systematically.

To make the connection with the JT model more precise, we need to be clearer about where the boundary of spacetime is located. To begin, the spacetime has a boundary at asymptotic infinity; it is at this boundary that the GH term and the additional boundary term involving the gauge field in eq. (2.29) are to be evaluated. However, in the JT model, the boundary is not at asymptotic infinity. It is located instead in the "asymptotic $\mathrm{AdS}_{2}$ " region. The two boundary terms, the GH term and the one involving the gauge field are evaluated at this boundary to obtain the action, eq. (2.31).

Let us now explain where the asymptotic $\mathrm{AdS}_{2}$ region lies in spacetime. From eq. (2.17) and eq. (2.30), we see that

$$
\phi=\frac{r-r_{h}}{r_{h}}=\frac{L_{2}^{2}}{r_{h}} \frac{1}{z}
$$


Comparing with eq. (2.5) we see therefore that the energy scale which characterises the breaking of scale invariance in the near-horizon region is

$$
\mathcal{J}=\frac{r_{h}}{L_{2}^{2}} \text {. }
$$

We are interested here in the behaviour at sufficiently small temperatures. At these low temperatures, the geometry has a region, which is sufficiently far from the horizon so that the effects of finite temperature have "died down", but not so far that the effects of the breaking of scale invariance due to the scale $\mathcal{J}$ have become significant. This is the asymptotic $\mathrm{AdS}_{2}$ region we referred to above. The boundary in the JT model we consider will be located in this asymptotic $\mathrm{AdS}_{2}$ region at a fixed value of $\phi$, eq. (2.2).

We can now be more precise about how low $T$ should be for our considerations to apply. We take $T$ to satisfy the condition,

$$
T \ll \mathcal{J}
$$

The $\mathcal{O}\left(z^{2}\right)$ corrections in eq. (2.6) for the black hole solutions are actually of order $(T z)^{2}$. The asymptotic $\mathrm{AdS}_{2}$ region then lies in the range where

$$
\frac{1}{\mathcal{J}} \ll z \ll \frac{1}{T} .
$$

The boundary which lies at $z_{B}$ also satisfies this condition. It follows then from the upper end of the inequality in eq. (2.38) that in this region the $\mathcal{O}\left(z^{2}\right)$ corrections to the metric are small, while it follows from the lower end that the boundary is still in the near-horizon region far from the asymptotically flat or $\mathrm{AdS}_{4}$ region of spacetime. These considerations were also discussed above around eq. (2.7). It is worth emphasising that our considerations apply to near-extremal black holes in asymptotically AdS and flat spacetimes, with the boundary of the $\mathrm{AdS}_{2}$ region satisfying eq. (2.38). The relation between the scale $\mathcal{J}$ and the underlying parameters varies depending on the kind of black hole being considered. For related comments, see section 3.1.1 of this paper and ref. [8].

Note that from eq. (2.35) and eq. (2.38) it also follows that

$$
\phi \ll 1
$$

in the region of spacetime extending from the horizon to the asymptotic $\mathrm{AdS}_{2}$ boundary, see eq. (2.7) and the comment thereafter. Away from the $\mathrm{AdS}_{2}$ boundary, $\phi$ no longer satisfies the condition eq. (2.39) and becomes large towards asymptotic infinity.

Comparing with the free energy for the JT model, eq. (2.14), and keeping track of the values for $\widetilde{G}$, and $\mathcal{J}$, eq. (2.33) and (2.36), we then get that the temperature dependence of the free energy for a near-extremal $\mathrm{RN}$ black hole is given by

$$
\Delta F=-\frac{2 \pi^{2} r_{h} L_{2}^{2}}{G_{4}} T^{2} .
$$

This agrees with the well-known result for a near-extremal RN black hole obtained using the full on-shell action, see eq. (B.13) (with $d=3$ ). 
Note that in the JT model, the action of the scaling symmetry of $\mathrm{AdS}_{2}$, under which the coordinates $(z, t) \rightarrow(\lambda z, \lambda t)$ takes $\mathcal{J} \rightarrow \mathcal{J} / \lambda$ and $T \rightarrow T / \lambda$. To get agreement with the black hole free energy, eq. (2.40), we need to fix the scale in the JT model to agree with the convention for temperature, $T$, at asymptotic infinity.

\section{A more detailed comparison with the JT model}

In this section, we explain why the JT model correctly gives the free energy for nearextremal black holes. Our explanation is remarkably general; it applies to the lowtemperature behaviour obtained from all near-extremal black holes which have a nearhorizon $\mathrm{AdS}_{2}$ region in their geometry. We start with considering the Reissner-Nordström black hole in this subsection, then analyse a more general theory involving scalars, gauge fields and gravity in the following subsection, and finally in subsection 3.3, consider rotating black holes.

One key point in the following discussion is as follows: the leading behaviour at low temperature is obtained by computing the on-shell action for a slightly non-extremal solution. This in turn is obtained by keeping the leading corrections in the solution above extremality. Now, away from the near-horizon region, these corrections can be treated as small perturbations and since the extremal black hole is also a solution to the equations of motion, the contribution due to these perturbations, at first order, reduces to the surface terms, eq. (3.9), at the boundary of the $\mathrm{AdS}_{2}$ region. In the near-horizon region, some of the corrections cannot be treated as perturbations, but here their contribution to the bulk action is correctly captured by the JT model action, eq. (2.1).

\subsection{RN black holes}

Let us now give more details for the RN case. Carrying out the dimensional reduction described above, we get the two-dimensional action, eq. (2.29). The resulting radial integral involved in eq. (2.29) extends from the horizon to asymptotic infinity; we can write this as a sum of two terms:

$$
I=I_{\left[H \rightarrow \partial \mathrm{AdS}_{2}\right]}+I_{\left[\partial \mathrm{AdS}_{2} \rightarrow \infty\right]} .
$$

Here $I_{\left[H \rightarrow \mathrm{AAdS}_{2}\right]}$ denotes the contribution from the horizon to the boundary of asymptotic $\mathrm{AdS}_{2}$ region described above while $I_{\left[\partial \mathrm{AdS}_{2} \rightarrow \infty\right]}$ denotes the contribution from the boundary of the asymptotic $\mathrm{AdS}_{2}$ region to asymptotic infinity. In particular, we take,

$$
\begin{aligned}
I_{\left[\partial \mathrm{AdS}_{2} \rightarrow \infty\right]}= & -\frac{1}{4 G_{4}} \int_{\partial \mathrm{AdS}_{2}}^{\infty} \mathrm{d}^{2} x \sqrt{g}\left(\Phi^{2} R+\frac{2 \Phi_{0}}{\Phi}-2 \Phi_{0} \Phi \Lambda_{4}+\frac{\Phi^{3}}{\Phi_{0}} F^{\alpha \beta} F_{\alpha \beta}\right) \\
& -\frac{1}{2 G_{4}} \int_{\infty} \mathrm{d} x \sqrt{\gamma}\left(\Phi^{2} K-\frac{2 \Phi^{3}}{\Phi_{0}} n_{\alpha} F^{\alpha \beta} A_{\beta}\right)+I_{\mathrm{CT}}^{\infty} \\
& -\frac{1}{G_{4}} \int_{\partial \mathrm{AdS}_{2}} \mathrm{~d} x \sqrt{\gamma} \frac{\Phi^{3}}{\Phi_{0}} n_{\alpha} F^{\alpha \beta} A_{\beta} .
\end{aligned}
$$

Here we have included the boundary terms in eq. (2.29) evaluated at infinity, as well as a counter-term $I_{C T}^{\infty}$, also to be evaluated at infinity, which is needed to get a finite result, as 
per the standard calculational procedure for computing the free energy. Finally, we have included an additional boundary term at the $\mathrm{AdS}_{2}$ boundary - this is the last term above involving the gauge field. In this term, the normal one-form $n_{\alpha}$ is taken to point from the $\mathrm{AdS}_{2}$ region towards asymptotic infinity. The contribution from the near-horizon region is given by

$$
\begin{aligned}
I_{\left[H \rightarrow \partial \mathrm{AdS}_{2}\right]}= & -\frac{1}{4 G_{4}} \int_{H}^{\partial \mathrm{AdS}_{2}} \mathrm{~d}^{2} x \sqrt{g}\left(\Phi^{2} R+\frac{2 \Phi_{0}}{\Phi}-2 \Phi_{0} \Phi \Lambda_{4}+\frac{\Phi^{3}}{\Phi_{0}} F^{\alpha \beta} F_{\alpha \beta}\right) \\
& +\frac{1}{G_{4}} \int_{\partial \mathrm{AdS}_{2}} \mathrm{~d} x \sqrt{\gamma} \frac{\Phi^{3}}{\Phi_{0}} n_{\alpha} F^{\alpha \beta} A_{\beta} .
\end{aligned}
$$

The bulk integral extends from the horizon to the $\mathrm{AdS}_{2}$ boundary and the additional boundary term exactly cancels the last term in eq. (3.2).

We will be interested in the contribution made in the on-shell action due to the finite temperature deformation of the solution, at leading order in $T$, keeping the charge $Q$ fixed. Consider first the near-horizon term, eq. (3.3). We denote the contribution this term makes by $\delta I_{\left[H \rightarrow \partial \mathrm{AdS}_{2}\right]}$. The dimensionless parameter characterising the solution is $T / \mathcal{J}$, where $\mathcal{J}$ is determined by the linear variation of the dilaton, eq. (2.5), eq. (2.36). Note that $T / \mathcal{J} \ll 1$ at low temperatures. The leading contribution in this parameter can be obtained by expanding the dilaton $\Phi$ in terms of the correction $\phi$, eq. (2.30), and only keeping terms which are of first order in $\phi$. This is because higher order terms will be suppressed by additional powers of $1 / \mathcal{J}$ and will therefore be small. This justifies why the last line in eq. (2.31), involving quadratic and higher powers of $\phi$ can be neglected. From the discussion in the previous section, it then follows that

$$
\delta I_{\left[H \rightarrow \partial \mathrm{AdS}_{2}\right]}=\delta I_{\mathrm{JT}}^{\mathrm{bulk}},
$$

where

$$
I_{\mathrm{JT}}^{\mathrm{bulk}}=-\frac{1}{16 \pi \widetilde{G}} \int \mathrm{d}^{2} x \sqrt{g} \phi\left(R-\Lambda_{2}\right),
$$

with $\widetilde{G}$ given by eq. (2.33).

Next consider the finite temperature contribution made from the region away from the $\mathrm{AdS}_{2}$ horizon, eq. (3.2), which we denote by $\delta I_{\left[\partial \mathrm{AdS}_{2} \rightarrow \infty\right]}$. A black hole at small temperature is given by the solution eq. (2.15) where $f(r)$ takes the form,

$$
f(r)=f_{\text {ext }}(r)+\delta f(r)
$$

where

$$
\begin{aligned}
f_{\mathrm{ext}}(r) & =\frac{\left(r-r_{h}\right)^{2}}{r^{2}}\left(1+\frac{r^{2}+2 r r_{h}+3 r_{h}^{2}}{L^{2}}\right), \\
\delta f(r) & =-\frac{2 G_{4} \delta M}{r}
\end{aligned}
$$

with $\delta M$ being the mass above extremality. In the region being considered here, $f_{\text {ext }}$ does not vanish and for small $\delta M, \delta f$ can be treated as a small perturbation, since $\delta f / f_{\text {ext }} \ll 1$. 
As was discussed above, the change in the action then comes from a surface term evaluated at the boundary of $\mathrm{AdS}_{2}$.

Some of the steps are described in appendix A. One finds that

$$
\delta I_{\left[\partial \mathrm{AdS}_{2} \rightarrow \infty\right]}=\delta I_{\mathrm{GH}}+\frac{1}{8 \pi \widetilde{G}} \int_{\partial \mathrm{AdS}_{2}} \mathrm{~d} x \delta(\sqrt{\gamma}) \phi K,
$$

where $I_{\mathrm{GH}}$ is given in eq. (2.11) and both terms are to be evaluated at the boundary of the asymptotic $\mathrm{AdS}_{2}$ region. Also, $\delta(\sqrt{\gamma})$ arises from the first order change in the boundary metric.

The reader will recall from our discussion of the previous section that the counterterm (2.12) is chosen to cancel the divergence in the GH term, eq. (2.11). As argued in appendix A, it then follows from the behaviour of the metric in the asymptotic $\mathrm{AdS}_{2}$ region that

$$
\frac{1}{8 \pi \widetilde{G}} \int_{\partial \mathrm{AdS}_{2}} \mathrm{~d} x \delta(\sqrt{\gamma}) \phi K=\frac{1}{8 \pi \widetilde{G} L_{2}} \int \mathrm{d} x \delta(\sqrt{\gamma}) \phi=\delta I_{\mathrm{CT}},
$$

where we have used eq. (2.12). From eq. (3.9), eq. (3.10) we learn that

$$
\delta I_{\left[\partial \mathrm{AdS}_{2} \rightarrow \infty\right]}=\delta I_{\mathrm{JT}}^{\text {boundary }}
$$

where

$$
I_{\mathrm{JT}}^{\text {boundary }}=-\frac{1}{8 \pi \widetilde{G}} \int_{\partial \mathrm{AdS}_{2}} \mathrm{~d} x \sqrt{\gamma} \phi\left(K-\frac{1}{L_{2}}\right) .
$$

Combining eq. (3.4) and eq. (3.11) we see that the full change in the action is correctly given by the JT model. Note that for establishing this result, it was important that the boundary conditions, eq. (2.2) and eq. (2.6) are met at the $\mathrm{AdS}_{2}$ boundary.

\subsubsection{Some comments}

A few comments are worth making at the end of this subsection. First, we note that for the asymptotically flat case, $r_{h}=Q \sim q^{\prime} / M_{\mathrm{Pl}}$ (where $q^{\prime}$ is the dimensionless charge carried by the black hole) and $L_{2}=r_{h}$; so that the condition eq. (2.37) becomes

$$
\operatorname{Tr}_{h} \sim \frac{T q^{\prime}}{M_{\mathrm{Pl}}} \ll 1
$$

In the asymptotically $\mathrm{AdS}_{4}$ case, on the other hand, for a big black hole with $r_{h} \gg L$, $L_{2}=L / \sqrt{6}$, while the chemical potential (as seen by the boundary CFT for example) is $\mu=r_{h} / L^{2} \sim r_{h} / L_{2}^{2}$. Thus eq. (2.37) can be stated as,

$$
T \ll \mu .
$$

Second, it is straightforward to generalise the discussion above to RN black holes in arbitrary dimensions. The action for the canonical partition function in this case is given by

$$
\begin{aligned}
I= & -\frac{1}{16 \pi G_{d+1}} \int \mathrm{d}^{d+1} x \sqrt{g}\left(R-2 \Lambda_{d+1}+F_{\mu \nu} F^{\mu \nu}\right) \\
& -\frac{1}{8 \pi G_{d+1}} \int \mathrm{d}^{d} x \sqrt{\gamma}\left(K-2 n_{\mu} F^{\mu \nu} A_{\nu}\right) .
\end{aligned}
$$


Performing a dimensional reduction by writing the metric as in eq. (B.14), and expanding about the attractor value for $\Phi$ gives a JT model, with $\widetilde{G}$ given in eq. (B.22), and $\mathcal{J}$ given by eq. (B.18). As a result, from the JT model, we get the free energy to be eq. (2.14) which, in fact, agrees with the result as obtained in eq. (B.13). See appendix B for more details.

Third, while it is intuitively clear that the low temperature thermodynamics should arise from the near-horizon region, and the well-known diverging back-reaction to perturbations in $\mathrm{AdS}_{2}$ [132] suggests that at least a linear variation of the dilaton must be incorporated, what the above argument makes precise is that to get agreement with the JT model, it is crucial that the boundary of the near-horizon region is located at a fixed value of the dilaton, eq. (2.2).

Finally, the agreement with the JT model for thermodynamics can also be extended for the response of the black hole to low-frequency time-dependent probes, as discussed in [7, 8]. ${ }^{1}$ Higher partial waves can also be systematically incorporated in the two-dimensional theory, and in fact, a precise version of the near- $\mathrm{AdS}_{2}$ near-CFT 1 correspondence can be then given for these systems [8].

\subsection{More general theories}

The arguments above, showing that the thermodynamics at low energies for near-extremal RN black holes is correctly obtained from the JT model, can be extended to more general theories involving additional scalars and gauge fields. Let us take a theory in $d+1$ dimensions consisting of $m$ gauge fields, $n$ scalars and gravity, with two-derivative interactions given by the action

$$
\begin{aligned}
I= & -\frac{1}{16 \pi G_{d+1}} \int \mathrm{d}^{d+1} x \sqrt{g}\left[R+\hat{f}_{a b}\left(\Psi_{i}\right) F_{a \mu \nu} F_{b}^{\mu \nu}+\hat{h}^{p q}\left(\Psi_{i}\right) g^{\mu \nu} \nabla_{\mu} \Psi_{p} \nabla_{\nu} \Psi_{q}+\hat{V}\left(\Psi_{i}\right)\right] \\
& -\frac{1}{8 \pi G_{d+1}} \int \mathrm{d}^{d} x \sqrt{\gamma} K .
\end{aligned}
$$

Here, the indices range from $a, b=1, \cdots, m$, and $p, q=1, \cdots, n, \hat{f}_{a b}$ are gauge kinetic energy coefficients, $\hat{h}_{p q}$ is a metric in the space of scalars and $\hat{V}$ is a potential. Repeated indices are summed over in eq. (3.16). In four dimensions, we can also have $F_{\mu \nu} \widetilde{F}^{\mu \nu}$ type of terms and the black holes can also carry magnetic charges. Similarly, in higher dimensions we can add higher form fields under which the black hole carries magnetic charges. We do not explicitly consider these possibilities below although they can be easily included.

To carry out a dimensional reduction, we take the metric to have the form

$$
\mathrm{d} s^{2}=\left(\frac{\Phi_{0}}{\Phi}\right)^{d-2} g_{\alpha \beta} \mathrm{d} x^{\alpha} \mathrm{d} x^{\beta}+\Phi^{2} \mathrm{~d} \Omega_{d-1}^{2},
$$

where $g_{\alpha \beta}$ refers to the metric in the radial and time directions This gives from eq. (3.16) a two-dimensional action,

$$
\begin{aligned}
I= & -\frac{v_{d-1}}{16 \pi G_{d+1}} \int \mathrm{d}^{2} x \sqrt{g}\left[\Phi^{d-1} R+V\left(\Psi_{i}, \Phi\right)+\right. \\
& f^{a b}\left(\Phi, \Psi_{i}\right) F_{a \alpha \beta} F_{b}^{\alpha \beta} \\
& \left.+h^{p q}\left(\Phi, \Psi_{i}\right) g^{\alpha \beta} \nabla_{\alpha} \Psi_{p} \nabla_{\beta} \Psi_{q}\right] \\
- & \frac{v_{d-1}}{8 \pi G_{d+1}} \int \mathrm{d} x \sqrt{\gamma} \Phi^{d-1} K+\frac{v_{d-1}}{4 \pi G_{d+1}} \int \mathrm{d} x \sqrt{\gamma} f^{a b}\left(\Phi, \Psi_{i}\right) n_{\alpha} F_{a}^{\alpha \beta} A_{b \beta} .
\end{aligned}
$$

\footnotetext{
${ }^{1}$ It is important to include the extra phase mode and its couplings to charged probes, which we have not described here.
} 
One scalar - the dilaton $\Phi$ is special; it appears in the coefficient of the Ricci scalar, $R$. $\Phi_{0}$ is its attractor value at the horizon. We have also added an extra boundary term dependent on the gauge field - the last one above - since we are interested in the free energy at fixed values of the electric charge. Here, $v_{d-1}$ denotes the volume of the unit $S^{d-1}$, eq. (B.7).

We are considering systems which have an extremal black hole solution with a nearhorizon $\mathrm{AdS}_{2} \times S^{d-1}$ region. The near-horizon region is well known to be an attractor in these cases. The gauge fields in this region take the values

$$
F_{a}=\frac{1}{2} f_{a b} Q^{b} \sqrt{g} \epsilon_{\alpha \beta} \mathrm{d} x^{\alpha} \wedge \mathrm{d} x^{\beta},
$$

where $Q^{b}$ are the charges carried by the black hole, $f_{a b}$ is the inverse of $f^{a b}$, and $\frac{1}{2} \sqrt{g} \epsilon_{\alpha \beta} \mathrm{d} x^{\alpha} \wedge \mathrm{d} x^{\beta}$ is the volume form in $\mathrm{AdS}_{2}$. The scalars in the near-horizon region take the attractor values $\left\{\Phi_{0}, \Psi_{0 i}\right\}$. The resulting attractor values for the scalars can be computed by extremising the entropy function $[133,134]$ or by using an effective potential [135].

The free energy is given by the on-shell value of this action evaluated for a black hole at temperature $T$ carrying charges $Q^{a}$. The action in eq. (3.18) is again a sum of two terms. One, involving the integral from the horizon to the asymptotic $\mathrm{AdS}_{2}$ region and the second, from the asymptotic $\mathrm{AdS}_{2}$ region to asymptotic infinity. We denote them, as above, by $I_{\left[H \rightarrow \partial \mathrm{AdS}_{2}\right]}$ and $I_{\left[\partial \mathrm{AdS}_{2} \rightarrow \infty\right]}$ respectively, these are analogous to eq. (3.3) and eq. (3.2) above.

In the near-horizon region of the extremal solution, once one is away from the horizon, the dilaton evolves with a linear radial dependence, e.g., eq. (B.17). Similarly, the other scalars $\Psi_{i}$ also evolve along the radial direction and their evolution gives rise to scales $\mathcal{J}_{i}$ which are analogous to the scale $\mathcal{J}$ that characterises the breaking of scale invariance due to the non-constant dilaton. We will take all these scales to be comparable to simplify the discussion, i.e., $\mathcal{J} \sim \mathcal{J}_{i}$. The condition for temperature $T$ to be low enough is then given by

$$
T \ll \mathcal{J}, \mathcal{J}_{i} \text {. }
$$

Expanding the scalars about their attractor values,

$$
\begin{aligned}
\Phi & =\Phi_{0}(1+\phi), \\
\Psi_{i} & =\Psi_{0 i}+\delta \Psi_{i} .
\end{aligned}
$$

we locate the boundary of the $\mathrm{AdS}_{2}$ region at a location where the dilaton takes a fixed value, $\phi_{B}$, with the condition, eq. (2.38), being met. The other scalars $\delta \Psi_{i}$ do not take fixed values at this boundary, but it follows from eq. (2.38), eq. (3.20) that their boundary values meet the condition, $\delta \Psi_{B i} / \Psi_{0 i} \ll 1$.

In the near-horizon region, for calculating the contribution $I_{\left[H \rightarrow \partial \mathrm{AdS}_{2}\right]}$ makes to the free energy, we can work to linear order in $\phi$ and $\delta \Psi$, since higher order terms will be suppressed by higher powers of $\mathcal{J}, \mathcal{J}_{i}$ and will thus be suppressed at low $T / \mathcal{J}$. It is then easy to see that the contribution of the near-horizon region is given by eq. (3.5) with $\widetilde{G}$ taking the value,

$$
\widetilde{G}=\frac{G_{d+1}}{v_{d-1}(d-1) \Phi_{0}^{d-1}}
$$


In the region away from the horizon, on the other hand, the leading order changes in the metric etc., can all be treated as small perturbations, as in the RN case. Thus the resulting contribution to $\delta I_{\left[\partial \mathrm{AdS}_{2} \rightarrow \infty\right]}$ comes from surface terms at the $\mathrm{AdS}_{2}$ boundary. Surface terms which arise from the curvature dependent term, $\Phi^{d-1} R$, in eq. (3.18) give rise, at linear order in $\phi$ to the boundary terms in the JT model, eq. (3.12), this follows from an argument analogous to the RN case discussed in appendix A. Surface terms arising from the scalar kinetic energy terms in eq. (3.18) do not lead to any contribution at leading order, as explained in appendix A. As a result, once again, we find that the JT model correctly gives the leading free energy at small $T$.

Let us end with some comments. It is easy to argue that the leading departure away from the attractor value for $\phi$ takes the form eq. (2.5), in fact this follows from the equations of motion in the JT model. $\mathcal{J}$ is now an energy scale that depends in general on all the charges $Q^{a}$, as well as the asymptotic data at infinity. Also, the resulting free energy is given by eq. (2.14) with $\widetilde{G}$ being given by eq. (3.22). Note that as was mentioned above, the other scalars also evolve and this gives rise to additional sources for the breaking of scale invariance; however quite notably, it is the breaking due to the evolution of the dilaton that determines the free energy.

\subsection{Rotating black holes}

The metric for a rotating black hole is well known to be quite complicated, as will also be apparent below. Despite these complications, quite interestingly, the discussion above can be extended to such black holes as well in a fairly straightforward way.

A few important features come to our aid in simplifying the analysis and connecting it to the JT model.

First, many rotating extremal black holes are also known to posses an $\mathrm{AdS}_{2}$ symmetry in their near-horizon geometry and our analysis applies only to such cases. In fact, this property is true quite generically for rotating black holes when their extremal entropy is non-vanishing. For example, for an extremal 4-dimensional Kerr black hole [136], in asymptotically flat spacetime, the near-horizon geometry is [137]

$$
\mathrm{d} s^{2}=\frac{1+\cos ^{2} \theta}{2}\left(\frac{r^{2}}{2 r_{h}{ }^{2}} \mathrm{~d} t^{2}+\frac{2 r_{h}^{2}}{r^{2}} \mathrm{~d} r^{2}+2 r_{h}^{2} \mathrm{~d} \theta^{2}\right)+\frac{4 r_{h}{ }^{2} \sin ^{2} \theta}{\left(1+\cos ^{2} \theta\right)}\left(\mathrm{d} \varphi-\mathrm{i} \frac{r}{2 r_{h}{ }^{2}} \mathrm{~d} t\right)^{2} .
$$

In these coordinates, the horizon is located at $r=0$ (the radius of the event horizon is $r_{h}$ in Boyer-Lindquist coordinates [138]; see below). This metric exhibits the $\mathrm{AdS}_{2}$ symmetries with the Killing vectors being

$$
\begin{aligned}
\zeta_{1} & =-L_{2} \frac{\partial}{\partial t} \\
\zeta_{0} & =t \frac{\partial}{\partial t}-r \frac{\partial}{\partial r} \\
\zeta_{-1} & =\frac{L_{2}}{2}\left(\frac{L_{2}^{2}}{r^{2}}-\frac{t^{2}}{L_{2}^{2}}\right) \frac{\partial}{\partial t}+\frac{t r}{L_{2}} \frac{\partial}{\partial r}+\mathrm{i} \frac{L_{2}^{3} \alpha}{r} \frac{\partial}{\partial \varphi} .
\end{aligned}
$$

These generate an $\mathrm{SL}(2, \mathbb{R})$ algebra. In this case, $L_{2}^{2}=2 r_{h}^{2}$ and $\alpha=1 / 2 r_{h}^{2}$.

Second, it has also been argued that rotating extremal black holes with such an $\mathrm{AdS}_{2}$ symmetry exhibit the attractor phenomenon which fixes their near-horizon geometry. The 
values of various scalars as well as the angular dependence in the metric are fixed by extremising the entropy function [139].

Finally, despite the fact that for the rotating case rotational invariance is broken and many more components of the metric are excited, a key feature that allows the connection with the JT model to be made for purposes of obtaining the free energy is the following. In calculating the leading order temperature dependence of the free energy, many alterations in the metric, in going from the extremal to slightly non-extremal case, can be regarded as small perturbations in the whole of spacetime extending from the horizon to asymptotic infinity. It follows then along the lines of the argument given above in the rotationally invariant case, that there is no change in the on-shell action at first order due to these alterations, since the extremal black hole we perturb about is already a solution of the equations of motion. Furthermore, it is sufficient to work to first order in these alterations for obtaining the leading temperature dependence. Therefore these changes in the metric, while going from the extremal to the slightly non-extremal case, can be neglected.

This observation leaves only a few remaining changes in the metric which we have to keep track of. Even for these, there is a further simplification. Consider computing the free energy by dividing the contribution to the on-shell action as coming from the region close to the horizon, and sufficiently far away from it, analogous to the two terms, eq. (3.1), above. Now even though some changes are not small in the near-horizon region, they are small perturbations in the region far away from the horizon. As a result, the contribution of this far region can be expressed as a surface term at the boundary of the near-horizon region. This finally only leaves the near-horizon region, but here, the $\mathrm{AdS}_{2}$ symmetry and the attractor behaviour mentioned above come to our aid and allow us to conclude that the JT model is correct!

Let us give some more details now. For concreteness, we consider the asymptotically flat 4-dimensional case considered above. The metric, for a general non-extremal Kerr black hole is

$$
\mathrm{d} s^{2}=\frac{\rho^{2} \Delta_{r}}{\Sigma} \mathrm{d} t^{2}+\frac{\rho^{2}}{\Delta_{r}} \mathrm{~d} r^{2}+\rho^{2} \mathrm{~d} \theta^{2}+\sin ^{2} \theta \frac{\Sigma}{\rho^{2}}(\mathrm{~d} \varphi+\mathrm{i} \omega \mathrm{d} t)^{2},
$$

where

$$
\begin{aligned}
\Delta_{r} & =r^{2}-2 G_{4} M r+a^{2}, \\
\rho^{2} & =r^{2}+a^{2} \cos ^{2} \theta, \\
\Sigma & =\left(r^{2}+a^{2}\right)^{2}-a^{2} \Delta_{r} \sin ^{2} \theta, \\
\omega & =\frac{2 G_{4} M a r}{\Sigma} .
\end{aligned}
$$

The various functions appearing above are obtained by taking the $L \rightarrow \infty, q_{e} \rightarrow 0, q_{m} \rightarrow 0$ limit of the functions in eqs. (5.4)-(5.9). At extremality, $\Delta_{r}$ has a second order zero at $r=r_{h}=a_{0}$. (We have denoted the extremal value of $a$ by $a_{0}$.) This gives the near-horizon metric in eq. (3.23) (with $\varphi \rightarrow \varphi-i \Omega_{H} t$, where $\Omega_{H}=\omega\left(r_{h}\right)$; see also appendix D). 
The metric, eq. (3.25) depends on $M$, the mass and $J$, the angular momentum. They are related through the parameter $a$,

$$
J=M a
$$

The extremal mass is given by,

$$
M_{0}=\frac{r_{h}}{G_{4}}=\frac{a_{0}}{G_{4}} .
$$

When we go to the slightly non-extremal case keeping $J$ fixed, $M$ changes and from eq. (3.30) $a$ changes as well. This results in all the functions which appear in eq. (3.25) being altered. However, for some of them the fractional change is small everywhere in spacetime outside the horizon. For instance, for $\rho^{2}$ we have from eq. (3.27) that

$$
\delta \rho^{2}=2 a_{0} \cos ^{2} \theta \delta a,
$$

so that $\frac{\delta \rho^{2}}{\rho^{2}} \ll 1$ everywhere.

In contrast, the change in $\Delta_{r}$ while being small in the region from the $\mathrm{AdS}_{2}$ boundary to infinity, is not small in the near-horizon region, since it vanishes at $r=r_{h}$ in the extremal case. Therefore, $\Delta_{r}$ is a function whose change we need to keep track of in going to the non-extremal case.

To proceed, let us write the metric in the form,

$$
\mathrm{d} s^{2}=\frac{\rho^{2} \Delta_{r}}{\Sigma} \mathrm{d} t^{2}+\frac{\rho^{2}}{\Delta_{r}} \mathrm{~d} r^{2}+\Phi^{2}\left(\frac{\rho^{2}}{\sqrt{\Sigma}}\right) \mathrm{d} \theta^{2}+\Phi^{2} \sin ^{2} \theta\left(\frac{\sqrt{\Sigma}}{\rho^{2}}\right)\left(\mathrm{d} \varphi-\mathrm{i} A_{t} \mathrm{~d} t\right)^{2} .
$$

Here,

$$
\begin{gathered}
\Phi^{2}=\sqrt{\Sigma}=\left(\left(r^{2}+a^{2}\right)^{2}-a^{2} \Delta_{r} \sin ^{2} \theta\right)^{1 / 2}, \\
A_{t}=\Omega_{H}-\omega,
\end{gathered}
$$

and we are working in the shifted azimuthal coordinate $\varphi \rightarrow \varphi-i \Omega_{H} t$. One advantage of parametrising the metric in this manner is that the volume of the internal two sphere spanned by $\theta, \varphi$ is now given by $4 \pi \Phi^{2}$ and therefore only dependent on $\Phi$, and manifestly independent of $\Sigma$. Although $\Phi$ is evidently a function of $\Sigma$, as defined above, when $\Phi$ and $\Sigma$ are regarded formally as independent quantities, the volume of the transverse space depends only on $\Phi$. We emphasise the role of $\Phi$ to make the connections with the previous discussion more transparent. As a result, after dimensional reduction to two dimensions, the dilaton field will be proportional to $\Phi^{2}$ (as in eq. (2.25)), and the variation of $\Phi$ from its attractor value (2.30) will be important in obtaining the thermodynamics. In contrast, by repeating the analysis above for $\rho^{2}$, now for the field $\Sigma$, it is easy to see that its change from the extremal value will not be important in obtaining the leading order change in the free energy. We also note that we will need to keep track of the change in $\Delta_{r}$, and $A_{t}$, when going to the non-extremal case, for computing the corrections to the free energy.

It is now easy to obtain the bulk action in the near-horizon region. The metric in this region can be written as

$$
\mathrm{d} s^{2}=\frac{1+\cos ^{2} \theta}{2} g_{\alpha \beta} \mathrm{d} x^{\alpha} \mathrm{d} x^{\beta}+\frac{1+\cos ^{2} \theta}{2} \Phi^{2} \mathrm{~d} \theta^{2}+\Phi^{2} \frac{2 \sin ^{2} \theta}{1+\cos ^{2} \theta}\left(\mathrm{d} \varphi-\mathrm{i} A_{\alpha} \mathrm{d} x^{\alpha}\right)^{2} .
$$

Here, $\alpha, \beta$ take values in $r, t$. 
Starting from the action

$$
I=-\frac{1}{16 \pi G_{4}} \int \mathrm{d}^{4} x \sqrt{g} R
$$

and inputting the metric eq. (3.36), we obtain,

$$
I=-\frac{1}{4 G_{4}} \int \mathrm{d}^{2} x \sqrt{g}\left(\Phi^{2} R+2(\nabla \Phi)^{2}+1+\frac{1}{2} \Phi^{4} F_{\alpha \beta} F^{\alpha \beta}\right)+\frac{1}{G_{4}} \int_{\partial} \mathrm{d} x \sqrt{\gamma} n^{\alpha} \Phi \nabla_{\alpha} \Phi,
$$

where $R$ which appears in eq. (3.38) is the Ricci curvature of the two-dimensional metric. This reduction, a special case of the reduction we have carried out in section 5, (zero electric and magnetic charges and zero cosmological constant limit) was also considered in [140].

We are interested in a rotating black hole of angular momentum $J$; the field strength in this case is given by

$$
F_{\alpha \beta}=\frac{2 J G_{4}}{\Phi^{4}} \sqrt{g} \epsilon_{\alpha \beta} .
$$

Expanding $\Phi$ as given in eq. (2.30), where

$$
\Phi_{0}=\sqrt{2 G_{4} J}
$$

and carrying out a change in frame

$$
g_{\alpha \beta} \rightarrow \frac{\Phi_{0}}{\Phi} g_{\alpha \beta}
$$

gives, for the action eq. (3.38),

$$
\begin{aligned}
I= & -\frac{\Phi_{0}^{2}}{2 G_{4}} \int \mathrm{d}^{2} x \sqrt{g} \phi\left(R-\Lambda_{2}\right)+\frac{3 \Phi_{0}^{2}}{4 G_{4}} \int_{\partial} \mathrm{d} x \sqrt{\gamma} n^{\alpha} \nabla_{\alpha} \phi \\
& -\frac{\Phi_{0}^{4}}{2 G_{4}} \int_{\partial} \mathrm{d} x \sqrt{\gamma}(1+5 \phi) n_{\alpha} F^{\alpha \beta} A_{\beta} .
\end{aligned}
$$

Here we have only included terms to linear order in $\phi$ and not included a topological term leading to the ground state entropy. We note that the bulk term above agrees with the JT model.

We had mentioned that the region between the $\mathrm{AdS}_{2}$ boundary and infinity contributes a surface term at the $\mathrm{AdS}_{2}$ boundary. We add to this the two surface terms in eq. (3.42). A convenient way of calculating the resulting total of all surface terms is the following. We have argued that the changes in the two-dimensional metric etc. that we are interested in cannot be regarded as perturbations in the $\mathrm{AdS}_{2}$ region. But we could consider other changes which would be perturbations in the whole of spacetime outside the horizon. For such changes, the total alteration in the action to first order in these perturbations from the near $\mathrm{AdS}_{2}$ region should also have been a surface term at the boundary of $\mathrm{AdS}_{2}$ which cancels the surface term which arises from the region between the $\mathrm{AdS}_{2}$ boundary and infinity. This along with general covariance in the two-dimensional theory allows us to obtain the surface terms. 
Let us denote by $I_{\left[H \rightarrow \partial \mathrm{AdS}_{2}\right]}$ the bulk term in eq. (3.42):

$$
I_{\left[H \rightarrow \partial \mathrm{AdS}_{2}\right]}=-\frac{\Phi_{0}^{2}}{2 G_{4}} \int \mathrm{d}^{2} x \sqrt{g} \phi\left(R-\Lambda_{2}\right) .
$$

The change in the $\mathrm{AdS}_{2}$ region bulk action for perturbations which satisfies the Dirichlet boundary condition for $\phi$ gives, (see appendix A)

$$
\delta I_{\left[H \rightarrow \partial \mathrm{AdS}_{2}\right]}=\frac{\Phi_{0}^{2}}{G_{4}} \int_{\partial} \mathrm{d} x \delta(\sqrt{\gamma} K) \phi-\frac{\Phi_{0}^{2}}{G_{4}} \int_{\partial} \mathrm{d} x \delta(\sqrt{\gamma}) \phi K .
$$

Here the extrinsic curvature is computed using a normal which is directed outward towards asymptotic infinity. As a result, the surface term arising from the bulk integral from the $\mathrm{AdS}_{2}$ boundary to infinity. which cancels this contribution is opposite in sign from that in eq. (3.44). We see that with this change in sign, this contribution then agrees with what is obtained from the boundary terms in the JT model, we have also used the argument in subsection 3.1, eq. (3.10) that the second term above equals the contribution from the counter-term.

Since the bulk term in eq. (3.42) also agrees with the JT model, it then follows that the change in the free energy will agree with the result in the JT model. Let us also note that the connection to the JT model is tied to the near horizon geometry exhibiting an $\mathrm{SL}(2, \mathbb{R})$ symmetry. This symmetry results in the metric in the $t, r$ plane being conformally $\mathrm{AdS}_{2}$, eq. (3.23), and allows the free energy calculation, after carrying out the integral in the $\theta, \varphi$ directions, to be related to that in the JT model.

The discussion above can be extended to other rotating black holes, including those with extra gauge fields and scalars as well. The crucial point is that the bulk action in the near-horizon region becomes that of the JT model. It then follows that the additional surface terms can be also obtained from the near-horizon region and agree with those in the JT model, eq. (3.12), as was illustrated in the Kerr black hole above.

Comparing with the 4-dimensional Kerr case, it is clear that for a general $d+1$ dimensional spacetime where we start with the action,

$$
I=-\frac{1}{16 \pi G_{d+1}} \int \mathrm{d}^{d+1} x \sqrt{g} R+\cdots,
$$

$\widetilde{G}$, eq. (2.1), in the JT model which arises, is given by eq. (3.22). Note that the volume of the transverse space spanned by the angular directions is

$$
V_{d-1}=v_{d-1} \Phi^{d-1}
$$

with $v_{d-1}$ being the volume of the unit $S^{d-1}$, eq. (B.7), and $\Phi_{0}$ appearing in eq. (3.22) is the attractor value for $\Phi$. As in the cases above, the role of the dilaton in the JT model is played by the linear correction to $\Phi$ when it is expanded about $\Phi_{0}$ as given in eq. (2.30). And the scale $\mathcal{J}$, eq. (2.5), is determined by the linear variation in $\phi$ away from the attractor value.

We end with some comments. First, for a Kerr-Newman type of black hole, one can consider starting with no angular momentum and then adding some rotation. For small 
angular momentum, it is easy to see that extra Kaluza-Klein (KK) modes, corresponding to higher partial waves along the transverse sphere, are turned on. In such cases a twodimensional reduction can be carried out in the region between the $\mathrm{AdS}_{2}$ boundary and infinity as well, retaining these extra modes, and the analysis for these cases can be mapped to that in section 3.2. Second, our arguments for rotating black holes are somewhat less straightforward than for the spherically symmetric ones in the previous subsections. We will consider several examples below and see that the JT model does correctly give the free energy for the rotating cases as well. This adds additional evidence in support of these arguments.

Finally, as a summary of this section we note that we have seen that the dilaton, in all cases including rotating ones, corresponds to the volume of the transverse sphere spanned by the angular directions, with $\Phi_{0}$ being related to the area of the horizon, by eq. (3.46),

$$
A_{H}=v_{d-1} \Phi_{0}^{d-1}
$$

Using eq. (3.22) and eq. (2.14) this allows us to express the free energy for near-extremal black holes in $d+1$ spacetime dimensions, as

$$
\Delta F=-(d-1) \pi S_{0} \frac{T^{2}}{\mathcal{J}}
$$

where $S_{0}=A_{H} / 4 G_{d+1}$ is the ground state entropy.

\section{Five-dimensional rotating black holes in asymptotically Anti-de Sitter spacetime}

We are now ready to consider some specific examples of rotating near-extremal black holes. The first case we consider in this section is that of a five-dimensional rotating black hole in asymptotically $\mathrm{AdS}_{5}$ spacetime. This example was studied in [86] where the connection with the JT model was also explored. We will see that this example can, in fact, be related to the discussion in section 3.2 .

We start with the Euclidean action,

$$
I=-\frac{1}{16 \pi G_{5}} \int \mathrm{d}^{5} x \sqrt{g}\left(R-2 \Lambda_{5}\right)-\frac{1}{8 \pi G_{5}} \int \mathrm{d}^{4} x \sqrt{\gamma} K
$$

The metric of the five-dimensional rotating black hole is given by, (see [86] and [141-143])

$$
\mathrm{d} s^{2}=\frac{\Delta}{\Xi} \exp \left(U_{2}-U_{1}\right) \mathrm{d} t^{2}+\frac{r^{2}}{\left(r^{2}+a^{2}\right) \Delta} \mathrm{d} r^{2}+\exp \left(-U_{1}\right) \mathrm{d} \Omega_{2}^{2}+\exp \left(-U_{2}\right)(\mathrm{d} \psi+A)^{2}
$$

where $\Delta, U_{1}, U_{2}$ are functions of $r$ and $A$ is a one-form (see appendix C). Note that the angular coordinate $\psi$ lies in the range $[0,4 \pi)$. 
For the extremal black hole the parameter $\Delta$ has a second order zero in $r$. The metric in the near-horizon limit takes the attractor value

$$
\begin{aligned}
\mathrm{d} s^{2}= & \frac{1}{2\left(1+\frac{r_{h}^{2}}{L^{2}}\right)}\left(\frac{\left(r-r_{h}\right)^{2}}{L_{2}^{2}} \mathrm{~d} t^{2}+\frac{L_{2}^{2}}{\left(r-r_{h}\right)^{2}} \mathrm{~d} r^{2}\right)+\frac{r_{h}^{2}}{2\left(1-\frac{2 r_{h}^{2}}{L^{2}}\right)} \mathrm{d} \Omega_{2}^{2} \\
& +\frac{r_{h}^{2}}{\left(1-\frac{2 r_{h}^{2}}{L^{2}}\right)^{2}}(\mathrm{~d} \psi+\bar{A})^{2},
\end{aligned}
$$

where,

$$
\bar{A}=\cos \theta \mathrm{d} \varphi-\mathrm{i}\left(1-\frac{2 r_{h}^{2}}{L^{2}}\right)\left(1+\frac{2 r_{h}^{2}}{L^{2}}\right)^{1 / 2} \frac{r-r_{h}}{r_{h}^{2}} \frac{1}{\left(1+\frac{r_{h}^{2}}{L^{2}}\right)} \mathrm{d} t
$$

Here $L$ is the $\mathrm{AdS}_{5}$ radius, (C.1), and we have transformed the angular coordinate, $\mathrm{d} \psi \rightarrow$ $\mathrm{d} \psi+k \mathrm{~d} t$, for a constant $k$. We see that in the $r-t$ plane, at constant values for the angular coordinates, it clearly exhibits an $\mathrm{AdS}_{2}$ geometry. The radius of $\mathrm{AdS}_{2}$ is given by,

$$
L_{2}^{2}=r_{h}^{2} \frac{\left(1+\frac{r_{h}^{2}}{L^{2}}\right)}{\left(1+\frac{4 r_{h}^{2}}{L^{2}}\right)} .
$$

Going to the slightly non-extremal case, keeping the angular momentum $J$, eq. (C.8) fixed, it is easy to calculate the entropy and the free energy as a function of temperature $T$. One finds that

$$
\Delta F \equiv F-F_{\text {extremal }}=-\frac{\pi}{4 \widetilde{G}} \frac{T^{2}}{\mathcal{J}} .
$$

We have given the result in terms of the parameters $\widetilde{G}, \mathcal{J}$ in view of the discussion that follows. These parameters take the values,

$$
\begin{aligned}
\widetilde{G} & =\frac{G_{5}\left(1-\frac{2 r_{h}^{2}}{L^{2}}\right)^{2}}{24 \pi^{2} r_{h}^{3}}, \\
\mathcal{J} & =\frac{3}{r_{h}} \frac{1+\frac{4 r_{h}^{2}}{L^{2}}}{1-\frac{r_{h}^{2}}{L^{2}}} .
\end{aligned}
$$

We can now compare this result with the JT model that we obtain for this black hole. It is useful to carry out the dimensional reduction in obtaining the two-dimensional theory here in two steps. (Note that a similar dimensional reduction was carried out in [86]). First, writing the metric as

$$
\mathrm{d} s_{(5)}^{2}=g_{\mu \nu}^{(4)} \mathrm{d} x^{\mu} \mathrm{d} x^{\nu}+\Sigma^{2}(\mathrm{~d} \psi+A)^{2},
$$

we carry out a dimensional reduction of the standard KK type over the $\psi$ direction. This gives rise to a 4-dimensional theory, consisting of the metric, $g_{\alpha \beta}$, a gauge field $A$, and 
the scalar $\Sigma$. This theory is of the kind considered in section 3.2. The action of the 4-dimensional theory is

$$
I=-\frac{1}{4 G_{5}} \int \mathrm{d}^{4} x \sqrt{g^{(4)}}\left(\Sigma R-\frac{1}{4} \Sigma^{3} F_{\mu \nu} F^{\mu \nu}-2 \Sigma \Lambda_{5}\right)-\frac{1}{2 G_{5}} \int \mathrm{d}^{3} x \sqrt{\gamma^{(3)}} \Sigma K
$$

(which is closely related to the type of action considered in section 3.2). The gauge field here carries one unit of magnetic charge and also electric charge which is related to the angular momentum carried by the five-dimensional black hole. It is clear from eq. (4.2) that the 4-dimensional metric obtained after KK reduction is rotationally symmetric.

To come down to two dimensions, we next take the four-dimensional metric to have the form

$$
\mathrm{d} s_{(4)}^{2}=\frac{1}{\Sigma \Phi^{3 / 2}} g_{\alpha \beta} \mathrm{d} x^{\alpha} \mathrm{d} x^{\beta}+\frac{\Phi^{3}}{8 \Sigma} \mathrm{d} \Omega_{2}^{2} .
$$

The indices $\alpha, \beta$ refer to the two-dimensional coordinates. Here $\Phi^{3}$ is related to the 3 volume $V_{3}$ spanned by the $\theta, \varphi, \psi$ directions as follows:

$$
V_{3}=v_{3} \Phi^{3}
$$

where $v_{3}$ is the volume of a unit $S^{3}$.

Inserting eq. (4.11) in eq. (4.10), with the gauge field ansatz,

$$
A_{\mu} \mathrm{d} x^{\mu}=\cos \theta \mathrm{d} \varphi+\mathrm{i} A_{\alpha} \mathrm{d} x^{\alpha}
$$

gives the two-dimensional action, eq. (C.22) of appendix C. This action is of the form given in eq. (3.18).

From eq. (4.2) we see that that

$$
\Phi^{3}=8 \exp \left(-U_{1}-\frac{1}{2} U_{2}\right)
$$

Its attractor value is given by,

$$
\Phi_{0}^{3}=\frac{4 r_{h}^{3}}{\left(1-\frac{2 r_{h}^{2}}{L^{2}}\right)^{2}} .
$$

Expanding $\Phi$ about the attractor value, eq. (2.30), we get that $\phi$ varies linearly as given in eq. (2.5) with $z$ being related to the radial coordinate $r$ in eq. (2.20), and $\mathcal{J}$ taking the value, eq. (4.8). From eq. (C.26) and eq. (2.1) we see that that $\widetilde{G}$ takes the value, eq. (4.7).

It then follows from eq. (2.14) that the free energy of the JT model is also given by eq. (4.6) and agrees with the result above. More details are given in appendix C.

Let us note that in [86], the authors in their analysis of the JT model found an ambiguity in various thermodynamic parameters which corresponds to a choice of scale in defining the temperature. We have chosen conventions here so that the temperature agrees with the definition at asymptotic infinity.

We end this section with two comments. First, the free energy eq. (4.6) depends on $r_{h}$ which in turn can be obtained in terms of the angular momentum from eq. (C.8), eq. (C.12), 
eq. (C.13). Second these black holes, for $L \neq \infty$ could suffer from a superradiant instability, as discussed in $[144,145]$, see also appendix C. The flat spacetime limit $L \rightarrow \infty$ does not have this instability.

\section{Four-dimensional charged rotating black holes}

Next we study four-dimensional rotating black holes. With the strength we have by now acquired, we might as well consider the most general case of a Kerr-Newman near-extremal black hole with both electric and magnetic charges in asymptotically AdS spacetime! The uncharged near-extremal Kerr black hole in flat spacetime is a special case which we will comment on in more detail later on in the section.

The Euclidean action is,

$$
I=-\frac{1}{16 \pi G_{4}}\left[\int \mathrm{d}^{4} x \sqrt{g}\left(R-2 \Lambda_{4}-\hat{F}_{\mu \nu} \hat{F}^{\mu \nu}\right)+2 \int \mathrm{d}^{3} x \sqrt{\gamma} K\right] .
$$

There is a well-known rotating black hole solution given by [121, 146, 147].

$$
\mathrm{d} s^{2}=\frac{\rho^{2} \Delta_{r} \Delta_{\theta}}{\Sigma} \mathrm{d} t^{2}+\frac{\rho^{2}}{\Delta_{r}} \mathrm{~d} r^{2}+\frac{\rho^{2}}{\Delta_{\theta}} \mathrm{d} \theta^{2}+\frac{\Sigma}{\rho^{2} \Xi^{2}} \sin ^{2} \theta(\mathrm{d} \varphi+\mathrm{i} \omega \mathrm{d} t)^{2} .
$$

where,

$$
\begin{aligned}
L & =\sqrt{-\frac{3}{\Lambda_{4}}}, \\
\Xi & =1-\frac{a^{2}}{L^{2}}, \\
\rho^{2} & =r^{2}+a^{2} \cos ^{2} \theta, \\
\Delta_{r} & =\left(r^{2}+a^{2}\right)\left(1+\frac{r^{2}}{L^{2}}\right)-2 m r+\left(q_{e}^{2}+q_{m}^{2}\right), \\
\Delta_{\theta} & =1-\frac{a^{2}}{L^{2}} \cos ^{2} \theta, \\
\Sigma & =\left(r^{2}+a^{2}\right)^{2} \Delta_{\theta}-a^{2} \Delta_{r} \sin ^{2} \theta, \\
\omega & =\frac{a \Xi\left[\left(a^{2}+r^{2}\right) \Delta_{\theta}-\Delta_{r}\right]}{\Sigma} .
\end{aligned}
$$

The gauge field is given by,

$$
\hat{A}=\frac{q_{e} r}{\rho^{2}}\left(\mathrm{i} \mathrm{d} t+\frac{a \sin ^{2} \theta}{\Xi} \mathrm{d} \varphi\right)+\frac{q_{m} \cos \theta\left(r^{2}+a^{2}\right)}{\Xi \rho^{2}}\left(\mathrm{~d} \varphi+\mathrm{i} \frac{a \Xi}{r^{2}+a^{2}} \mathrm{~d} t\right) .
$$

The ADM mass, the angular momentum, the physical electric and magnetic charge are given by,

$$
\begin{aligned}
M & =\frac{m}{G_{4} \Xi^{2}}, \\
J & =\frac{m a}{G_{4} \Xi^{2}}, \\
Q_{e} & =\frac{q_{e}}{\Xi}, \\
Q_{m} & =\frac{q_{m}}{\Xi},
\end{aligned}
$$

respectively. 
Note that as in the five-dimensional case, (see appendix C) $|a|$ can approach $L$ only from below.

As discussed in appendix D, close to extremality, the change in entropy, above its extremal value, $\Delta S$ is given by

$$
\Delta S=\frac{\pi}{2 \widetilde{G}} \frac{T}{\mathcal{J}}
$$

where we have defined

$$
\widetilde{G}=G_{4} \frac{\left(1-\frac{a_{0}^{2}}{L^{2}}\right)}{8 \pi\left(r_{h}^{2}+a_{0}^{2}\right)},
$$

and

$$
\mathcal{J}=\frac{1}{r_{h}}\left(1+\frac{6 r_{h}^{2}+a_{0}^{2}}{L^{2}}\right)
$$

with an eye towards the discussion of the JT model that follows. Here, $r_{h}$ is the radial location of the extremal horizon and quantities with a subscript 0 denote the values of these parameters at extremality. For more details, see appendix D. Using thermodynamic relations, we then get the free energy above extremality to be,

$$
\Delta F=-\frac{\pi}{4 \widetilde{G}} \frac{T^{2}}{\mathcal{J}}
$$

This result can also be obtained directly by calculating the on-shell action.

Let us now connect this discussion to the JT model. Our discussion will follow section 3.3. The near-horizon metric for the extremal case is given by

$$
\begin{aligned}
\mathrm{d} s^{2}= & \frac{\rho_{0}^{2}}{\left(r_{h}^{2}+a_{0}^{2}\right)}\left(\frac{\left(r-r_{h}\right)^{2}}{L_{2}^{2}} \mathrm{~d} t^{2}+\frac{L_{2}^{2}}{\left(r-r_{h}\right)^{2}} \mathrm{~d} r^{2}\right)+\frac{\rho_{0}^{2} \Xi_{0}}{\left(r_{h}^{2}+a_{0}^{2}\right) \Delta_{\theta 0}} \Phi_{0}^{2} \mathrm{~d} \theta^{2} \\
& +\frac{\Delta_{\theta 0}\left(r_{h}^{2}+a_{0}^{2}\right)}{\Xi_{0} \rho_{0}^{2}} \Phi_{0}^{2} \sin ^{2} \theta\left(\mathrm{d} \varphi-\mathrm{i} \frac{2 r_{h} a_{0} \Xi_{0}}{\left(r_{h}^{2}+a_{0}^{2}\right)^{2}}\left(r-r_{h}\right) \mathrm{d} t\right)^{2}
\end{aligned}
$$

For further details, including the definitions of the various quantities appearing above, see appendix D. For the uncharged, asymptotically flat case, with $q_{e}, q_{m}=0, L \rightarrow \infty$, this agrees with eq. (3.23). The gauge fields in the near-horizon region takes the form given below in eq. (5.24) with

$$
\begin{array}{rlrl}
\mathcal{A}_{t} & =\frac{q_{e 0}\left(r_{h}^{2}-a_{0}^{2}\right)}{\left(r_{h}^{2}+a_{0}^{2}\right)^{2}}\left(r-r_{h}\right), & \mathcal{A}_{r}=0, \\
A_{t}=\frac{2 r_{h} a_{0} \Xi_{0}}{\left(r_{h}^{2}+a_{0}^{2}\right)^{2}}\left(r-r_{h}\right), & A_{r}=0 .
\end{array}
$$

The reader will note that the metric has an $\mathrm{AdS}_{2}$ factor, in the $t, r$ plane and an $\mathrm{SL}(2, \mathbb{R})$ symmetry with the generators given in eq. (3.24) with $L_{2}$ given by eq. (D.21) and

$$
\alpha=\frac{2 r_{h} a_{0} \Xi_{0}}{\left(r_{h}^{2}+a_{0}^{2}\right)^{2}} .
$$

The gauge field eq. (5.20) preserves the $\mathrm{SL}(2, \mathbb{R})$ symmetry. 
As discussed in section 3.3, to calculate the leading dependence of the free energy at small temperatures, we need to keep the change, due to non-extremality, in only some of the functions which appear in the metric, eq. (5.2). For example, the fractional change in $\rho^{2}$ is small in the whole of spacetime outside the horizon, similarly the change in $\Delta_{\theta}$ and $\Xi$. Thus the change in these function can be neglected and in the near-horizon region, we can take these function to have the form they do in the attractor geometry.

With these observations in mind, we now take the near-horizon metric to have the form,

$$
\mathrm{d} s^{2}=\frac{\rho_{0}^{2}}{\left(r_{h}^{2}+a_{0}^{2}\right)}\left(\frac{\Phi_{0}}{\Phi} g_{\alpha \beta} \mathrm{d} x^{\alpha} \mathrm{d} x^{\beta}+\frac{\Phi^{2} \Xi_{0}}{\Delta_{\theta 0}} \mathrm{~d} \theta^{2}\right)+\frac{\Delta_{\theta 0}\left(r_{h}^{2}+a_{0}^{2}\right)}{\rho_{0}^{2} \Xi_{0}} \Phi^{2} \sin ^{2} \theta\left(\mathrm{d} \varphi-\mathrm{i} A_{\alpha} \mathrm{d} x^{\alpha}\right)^{2},
$$

and the gauge field to be of the form,

$$
\hat{A}_{\mu} \mathrm{d} x^{\mu}=-\mathrm{i}\left(\mathcal{A}_{\alpha}+b(\theta) A_{\alpha}\right) \mathrm{d} x^{\alpha}+b(\theta) \mathrm{d} \varphi
$$

where,

$$
b(\theta)=\frac{1}{\Xi_{0} \rho_{0}^{2}}\left(q_{e 0} a_{0} r_{h} \sin ^{2} \theta+q_{m 0}\left(r_{h}^{2}+a_{0}^{2}\right) \cos \theta\right) .
$$

Here, the fields $\Phi, A_{\alpha}, \mathcal{A}_{\alpha}$ are assumed to be dependent only on the 2-dimensional coordinates $x^{\alpha}$. Note that volume of the two-sphere spanned by $\theta, \varphi$ is given by

$$
V_{2}=4 \pi \Phi^{2}
$$

$\Phi_{0}$ appearing in eq. (5.23) is the attractor value of $\Phi$.

Inserting in the action (5.1) and carrying out the integral over $\theta, \varphi$ gives the twodimensional action which agrees with the JT model, as is discussed in detail in appendix D.

Here let us summarise the key point in the analysis in appendix D. The 2-dimensional action which is obtained, eq. (D.26), has the form,

$$
I=-\frac{1}{4 G_{4}} \int \mathrm{d}^{2} x \sqrt{g} \Phi^{2} R+\cdots .
$$

Expanding $\Phi$, eq. (2.30) then gives from the term linear in $\phi$ to be of the form eq. (2.1) with $\widetilde{G}$ given in eq. (5.16). From the expansion for $\Phi$ defined in eq. (D.42), we also learn that $\mathcal{J}$ is given by eq. (5.17). It then follows from the analysis in the JT model that the free energy, eq. (2.14) agrees with the result obtained above, eq. (5.18).

\subsection{The near-extremal Kerr black hole}

The asymptotically flat uncharged Kerr black hole is an important special case. This is obtained by taking $L \rightarrow \infty$ and setting $q_{e}=q_{m}=0$ in all the formulae in the previous section.

The parameter $\mathcal{J}$, eq. (5.17), takes the value

$$
\mathcal{J}=\frac{1}{r_{h}} .
$$

and, from eq. (5.16),

$$
\widetilde{G}=\frac{G_{4}}{16 \pi r_{h}^{2}}
$$


In this case, $a_{0}=r_{h}$, eq. (D.7), and $r_{h}$ is related to the angular momentum of the black hole $J$ by

$$
r_{h}=\sqrt{G_{4} J} .
$$

Note that $r_{h}$ is of order the size of the event horizon.

The attractor values for $\Phi$, eq. (D.23) becomes,

$$
\Phi_{0}=\sqrt{2} r_{h}
$$

and $\phi$ varies as given in eq. (2.5) with $\mathcal{J}$ taking the value, eq. (5.28).

The dimensional reduction discussion goes through as described above, resulting in a two-dimensional action of the form

$$
I_{\mathrm{JT}}=-\frac{1}{16 \pi \widetilde{G}}\left(\int \mathrm{d}^{2} x \sqrt{g} \phi\left(R+\frac{2}{L_{2}^{2}}\right)+2 \int_{\partial} \mathrm{d} x \sqrt{\gamma} \phi K\right) .
$$

with $\widetilde{G}$ given in eq. (5.29).

The condition for the temperature to be small, eq. (2.37), using eq. (5.28), becomes,

$$
T \ll \mathcal{J}=\frac{1}{r_{h}} .
$$

We see that the temperature has to be much smaller than the inverse "light crossing time of the horizon".

From eq. (5.28) and eq. (5.29), we see that the resulting free energy at small temperatures is

$$
F=-\frac{4 \pi^{2} r_{h}^{3}}{G_{4}} T^{2}=-2 \pi S_{0} \sqrt{G_{4} J} T^{2}=-2 \pi S_{0} \frac{T^{2}}{\mathcal{J}},
$$

with $J$ being the angular momentum and $S_{0}$ being the zero-temperature entropy. This agrees with eq. (3.48) with $d=3$, see also [140].

Note also that the chemical potential which is the thermodynamic conjugate of $J$ is given for an extremal Kerr black hole by, eq. (D.4),

$$
\Omega_{H}=\frac{1}{2 a_{0}}
$$

while the angular momentum is given by, eq. (5.30)

$$
J=\frac{a_{0}^{2}}{G_{4}}
$$

This implies that the "charge susceptibility" [31] at zero temperature is given by

$$
\frac{\mathrm{d} J}{\mathrm{~d} \Omega_{H}}=-\frac{4 a_{0}^{3}}{G_{4}}=-4 \sqrt{G_{4} J^{3}}
$$

We had mentioned above in passing that an extra phase mode enters in the 2dimensional theory when one allows the charge to vary. It has an action schematically of the form, $\int \mathrm{d} \tau \mathcal{I} \dot{\theta}^{2}$. The susceptibility fixes the parameter $\mathcal{I}$, which acts like the moment of inertia of the phase mode. 


\subsection{Superradiant instabilities}

Let us also briefly discuss superradiant instabilities which can occur in the asymptotic antide Sitter spacetime [148-150]; a discussion for the 5-dimensional case is in appendix C; see also the end of section 4. A necessary condition for the superradiant instability to occur for black holes in asymptotically $\mathrm{AdS}_{4}$ spacetime is [144, 150],

$$
\Omega_{H}^{\infty}>\frac{1}{L}
$$

or equivalently, from eq. (D.4),

$$
r_{+}^{2}<a L
$$

Note that the angular velocity $\Omega_{H}^{\infty}$ is the thermodynamical variable conjugate to the angular momentum $J$. From eq. (D.7) it is easy to see that the inequality eq. (5.39), is always met for uncharged rotating extremal black holes $\left(q_{e}=q_{m}=0\right)$. However, it is possible to evade this bound for extremal black holes with sufficient amount of charge (see e.g., [151] for related discussion).

An extremal black hole is specified by three parameters, $q_{e}, q_{m}, a$ with the parameter $a$ satisfying the condition,

$$
a^{2}<L^{2}
$$

The radius of the horizon, $r_{h}$, is obtained from eq. (D.7) and given by

$$
r_{h}^{2}=\frac{-\left(L^{2}+a_{0}^{2}\right)+\sqrt{\left(L^{2}+a_{0}^{2}\right)^{2}+12 L^{2}\left(a_{0}^{2}+q_{0}^{2}\right)}}{6} .
$$

To evade the superradiant instability for the extremal case, one needs,

$$
r_{h}^{2}>a_{0} L
$$

In the supersymmetric case [152], (see also [153]), $q_{0}$ is determined in terms of $a_{0}$ by

$$
q_{0}^{2}=a_{0} L\left(1+\frac{a_{0}}{L}\right)^{2}
$$

One finds from eq. (5.41) that in this case $r_{h}^{2}=a_{0} L$ and one is at the edge of meeting the condition, eq. (5.42). By increasing $q_{0}$ and making it bigger than the SUSY value, eq. (5.43), for fixed $a_{0}$, it is easy to see from eq. (5.41) that $r_{h}$ increases and thus eq. (5.42) is now met.

In the SUSY case, if one makes the black hole slightly non-extremal, keeping the charges fixed at the SUSY values, one finds that the inequality eq. (5.39) is now violated and thus the superradiant instability goes away.

\section{Conclusions}

In this paper, we have argued that the low-temperature free energy for near-extremal black holes is correctly obtained from the JT model in general. In particular, our arguments apply for rotating black holes as discussed in section 3.3. These conclusion holds for all cases 
where the extremal geometry has an $\mathrm{AdS}_{2}$ factor and the related $\mathrm{SL}(2, \mathbb{R})$ symmetry. We have also verified by explicit calculations that these arguments are correct for near-extremal Kerr black hole in 5 dimensions and for a general Kerr-Newman black hole in 4 dimensions.

The dilaton field in the JT model corresponds to the volume of the transverse sphere spanned by the angular direction. It follows from our analysis that the temperature dependence of the free energy for all near-extremal black holes, including rotating ones, is given in a compact form by eq. (3.48) and determined by the ground state entropy and the scale $\mathcal{J}$ which characterises how the dilaton approaches its attractor value at the horizon.

The expectation that the JT model should be a good approximation, at sufficiently low temperatures and low frequencies for rotating black holes, which have an $\mathrm{AdS}_{2}$ factor in their near-horizon geometry, already arises from previous work [8, 139]. In [139], it was argued that extremal rotating black holes would also exhibit the attractor behaviour. One way to understand this is to construct the two-dimensional theory obtained after reducing over the angular direction. This theory contains the two-dimensional metric, the dilaton, which is the volume of the transverse sphere and appears as the coefficient of the EinsteinHilbert term in the two-dimensional theory, several gauge fields, scalars and also massive symmetric rank two tensor fields. In the rotating case, some of the gauge fields which are excited arise from components of the higher dimensional metric and similarly some of the scalars and the massive symmetric rank two fields arise from partial waves on the transverse sphere - these are excited due to the rotation. However, despite these complications, the analysis in the resulting two-dimensional theory is similar in the rotating and non-rotating cases, and all fields are drawn to their attractor values which can be obtained by extremising an entropy function.

The analysis of [139] was extended in [8] to include the slightly non-extremal case. It was argued that while allowing the dilaton to vary from its attractor value is important for controlling the back reaction, the departure for many other fields could be neglected to leading order, at low frequencies and low temperatures. This gives rise to a theory consisting of the JT model coupled to extra phase modes which arise from the gauge fields present in the two-dimensional theory. It follows from the arguments in [8] that this theory should be a good approximation for the low-frequency and low-temperature response of a near-extremal black hole. While rotating black holes were not explicitly considered in [8], the arguments there were based on the two-dimensional theory obtained after dimensional reduction over the transverse sphere and should apply for the rotating case as well, since as mentioned above, the resulting two-dimensional theories are similar.

In this paper, we have focussed on the low-temperature behaviour for near-extremal rotating black holes and confirmed both through a general argument and also explicit checks that this expectation is correct and that the JT model does indeed correctly account for the low-temperature free energy of such rotating near-extremal black holes.

These developments hold out the exciting possibility that the JT model could, in fact, be useful in analysing the dynamics of fast-spinning near-extremal black holes found in nature! In the paper, we saw that the thermodynamics of the black hole is correctly reproduced if the dilaton satisfies Dirichlet boundary conditions at the boundary of the $\mathrm{AdS}_{2}$ region. From the point of view of the four-dimensional metric, this means taking 
the volume of the transverse sphere to be constant along the boundary. Therefore, these results suggest that the dynamics of near-extremal rotating black holes, at sufficiently low frequencies but not necessarily small amplitudes, could be well approximated by an effective theory in which the near-horizon $\mathrm{AdS}_{2}$ region is replaced by a boundary located along the locus where the transverse sphere takes a constant volume. This boundary would interact with the stress tensor averaged over the transverse sphere and the dynamics of the black hole would then arise through these from interactions of the boundary. ${ }^{2}$

To get a feel for some of the numbers involved, consider a solar mass extremal Kerr black hole. It has a horizon of approximately $3 \mathrm{~km}$, with a light crossing time of $10^{-5} \mathrm{sec}$. Thus, one would expect that the JT model could be a good approximation for dynamics at frequencies which are smaller than about $10^{5} \mathrm{~Hz}$. Some examples of fast-spinning black holes are well known with masses in the range of a few solar masses. For example, GRS $1915+105$ involves a binary system with a black hole of mass $\sim 10 M_{\odot}$ and a spin parameter which has been measured to be around 0.98 [154-156] — making it very nearly extremal.

We hope to develop this connection with astrophysical fast-spinning black holes further in subsequent work.

The analysis in this paper and also in [7] and [8], suggest that one should be able to formulate a version of the fluid-gravity correspondence for near-extremal black holes including rotating ones. ${ }^{3}$ Consider a near-extremal black hole in asymptotically AdS spacetime with temperature $T \ll \mu$, where $\mu$ is the chemical potential corresponding to the charge carried by the black hole. For situations where $L^{-1} \ll T$, with $L$ being the length or time scale over which the fluid in the boundary theory varies, it is well known [157] that Einstein's equations lead to Navier-Stokes equations in the boundary theory. One might expect that when the variation is more rapid and satisfies the condition $T \ll L^{-1} \ll \mu$, also there is an effective theory that describes the dynamics, and it is obtained by coupling the JT model at the boundary of $\mathrm{AdS}_{2}$ in a suitable way with the outside. We hope to develop this theme in a subsequent paper as well.

The universal manner in which the JT model describes near-extremal black holes also allows for a fairly precise near- $\mathrm{AdS}_{2} /$ near-CFT 1 correspondence to be set up [8]. It will be very interesting to test this correspondence in examples where the extremal black holes arise in in string theory, with a near- $\mathrm{CFT}_{1}$ which can be understood in a microscopically precise way. The fact that rotating black holes are included in these examples, enlarges the set of possibilities even further. It might well be that some instances where this correspondence can be tested and understood most fruitfully involve rotating black holes.

\section{Acknowledgments}

We thank Indranil Halder and Shiraz Minwalla, for insightful discussions and comments. We thank the DAE, Government of India, for support. SPT also acknowledges support from

\footnotetext{
${ }^{2}$ As extra phase mode would also enter in the boundary action, since the in falling matter could change the angular momentum of the black hole. Its moment of inertia is determined by the charge susceptibility given in eq. (5.37).

${ }^{3}$ We are grateful to "The Professor" - Shiraz Minwalla - for his comments in this regard.
} 
the J. C. Bose fellowship of the DST, Government of India. UM thanks the Israel Institute for Advanced Studies, The Hebrew University of Jerusalem, Israel (The 36th Advanced School in Physics on Recent Progress in Quantum Field/String Theory) and the Abdus Salam International Centre for Theoretical Physics, Italy (Spring School on Superstring Theory and Related Topics) and the other organisers of these programmes for their warm hospitality during different stages of this work. We also acknowledge support from the Infosys Endowment for Research on the Quantum Structure of Spacetime. Most of all, we thank the people of India for generously supporting research in String Theory.

\section{A On how the Jackiw-Teitelboim model correctly reproduces the leading thermodynamic behaviour}

In this appendix, we elaborate on the comments made in section 3 and show exactly how the evaluation of the Euclidean on-shell action in the region far from the horizon (the bulk integral extending from the $\mathrm{AdS}_{2}$ boundary to the asymptotic infinity) gives rise to the Gibbons-Hawking boundary term and the counter-term in the Jackiw-Teitelboim model which are precisely the terms contributing to the JT on-shell action. To begin we consider the RN case studied in 3.1. The integral of interest in given in eq. (3.2),

$$
\begin{aligned}
I_{\left[\partial \mathrm{AdS}_{2} \rightarrow \infty\right]}= & -\frac{1}{4 G_{4}} \int_{\partial \mathrm{AdS}_{2}}^{\infty} \mathrm{d}^{2} x \sqrt{g}\left(\Phi^{2} R+\frac{2 \Phi_{0}}{\Phi}-2 \Phi_{0} \Phi \Lambda+\frac{\Phi^{3}}{\Phi_{0}} F^{\alpha \beta} F_{\alpha \beta}\right) \\
& -\frac{1}{2 G_{4}} \int_{\infty} \mathrm{d} x \sqrt{\gamma}\left(\Phi^{2} K-\frac{2 \Phi^{3}}{\Phi_{0}} n_{\alpha} F^{\alpha \beta} A_{\beta}\right)+I_{\mathrm{CT}}^{\infty} \\
& -\frac{1}{G_{4}} \int_{\partial \mathrm{AdS}_{2}} \mathrm{~d} x \sqrt{\gamma} \frac{\Phi^{3}}{\Phi_{0}} n_{\alpha} F^{\alpha \beta} A_{\beta} .
\end{aligned}
$$

We emphasise again that the normal one-form $n_{\alpha}$ appearing in the last line above (and the discussion below) points from the $\mathrm{AdS}_{2}$ boundary to the asymptotic infinity.

The change in $I_{\left[\partial \mathrm{AdS}_{2} \rightarrow \infty\right]}$ to first order in the metric, dilaton and gauge field perturbations, which we denote by, $\delta g_{\alpha \beta}, \delta \Phi$ and $\delta A_{\alpha}$, respectively, is given by,

$$
\begin{aligned}
\delta I_{\left[\partial \mathrm{AdS}_{2} \rightarrow \infty\right]}= & -\frac{1}{4 G_{4}} \int_{\partial \mathrm{AdS}_{2}} \mathrm{~d} x \sqrt{\gamma}\left(\Phi^{2} n_{\beta} \nabla_{\alpha} \delta g^{\alpha \beta}-\Phi^{2} g_{\alpha \beta} n^{\kappa} \nabla_{\kappa} \delta g^{\alpha \beta}\right) \\
& +\frac{1}{4 G_{4}} \int_{\partial \mathrm{AdS}_{2}} \mathrm{~d} x \sqrt{\gamma} \delta g^{\alpha \beta}\left(n_{\beta} \nabla_{\alpha} \Phi^{2}-g_{\alpha \beta} n^{\kappa} \nabla_{\kappa} \Phi^{2}\right) \\
& -\frac{1}{G_{4}} \int_{\partial \mathrm{AdS}_{2}} \mathrm{~d} x \delta\left(\sqrt{\gamma} \frac{\Phi^{3}}{\Phi_{0}} n_{\alpha} F^{\alpha \beta}\right) A_{\beta},
\end{aligned}
$$

where we have used the equations of motion, and also the fact that all fields vanish sufficiently fast at infinity. Note that all the three terms are surface terms at the boundary of $\mathrm{AdS}_{2}$, in agreement with the discussion in section 3. Note also that the quantity that is being varied in the third line of eq. (A.2) above is proportional to the charge carried by the black hole (see eq. (2.27)). Since we are interested in perturbations which do not change this charge, the term in the last line of eq. (A.2) vanishes. 
In order to obtain the first two lines of eq. (A.2), we have used the Palatini identity,

$$
\delta R_{\alpha \beta}=\nabla_{\kappa} \delta \Gamma_{\alpha \beta}^{\kappa}-\nabla_{\alpha} \delta \Gamma_{\beta \kappa}^{\kappa},
$$

where the variation of the Christoffel connection is given by,

$$
\delta \Gamma_{\alpha \beta}^{\lambda}=\frac{1}{2} g^{\lambda \kappa}\left(\nabla_{\alpha} \delta g_{\beta \kappa}+\nabla_{\beta} \delta g_{\alpha \kappa}-\nabla_{\kappa} \delta g_{\alpha \beta}\right) .
$$

The covariant differentiation and rearrangement of indices above are assumed to be done with the unperturbed metric.

As discussed in section 2, for obtaining the leading dependence of the action on temperature, it is enough to consider only terms up to linear order in $\phi$, eq. (2.30) at the boundary of $\mathrm{AdS}_{2}$. Expanding eq. (A.2) up to linear order in $\phi$ we get,

$$
\begin{aligned}
\delta I_{\left[\partial \mathrm{AdS}_{2} \rightarrow \infty\right]}= & -\frac{1}{16 \pi \widetilde{G}} \int_{\partial \mathrm{AdS}_{2}} \mathrm{~d} x \sqrt{\gamma}\left(\phi n_{\beta} \nabla_{\alpha} \delta g^{\alpha \beta}-\phi g_{\alpha \beta} n^{\kappa} \nabla_{\kappa} \delta g^{\alpha \beta}\right) \\
& +\frac{1}{16 \pi \widetilde{G}} \int_{\partial \mathrm{AdS}_{2}} \mathrm{~d} x \sqrt{\gamma} \delta g^{\alpha \beta}\left(n_{\beta} \nabla_{\alpha} \phi-g_{\alpha \beta} n^{\kappa} \nabla_{\kappa} \phi\right) .
\end{aligned}
$$

Here we have dropped some terms coming from the first line on the r.h.s. of eq. (A.2) which are independent of $\phi$ and turn out to vanish.

Let us now look at the Gibbons-Hawking boundary term on the $\mathrm{AdS}_{2}$ boundary, given by,

$$
I_{\mathrm{GH}}=-\frac{1}{8 \pi \widetilde{G}} \int_{\partial \mathrm{AdS}_{2}} \mathrm{~d} x \sqrt{\gamma} \phi K
$$

Its variation gives,

$$
\delta I_{\mathrm{GH}}=-\frac{1}{8 \pi \widetilde{G}} \int_{\partial \mathrm{AdS}_{2}} \mathrm{~d} x \delta(\sqrt{\gamma}) \phi K-\frac{1}{8 \pi \widetilde{G}} \int_{\partial \mathrm{AdS}_{2}} \mathrm{~d} x \sqrt{\gamma} \phi \delta K
$$

Note that, crucially, we have imposed a strict Dirichlet boundary condition on $\phi$ on the $\mathrm{AdS}_{2}$ boundary. We now use the relation that,

$$
\delta K=\nabla_{\alpha} \delta n^{\alpha}-\frac{1}{2} n^{\alpha} g_{\kappa \lambda} \nabla_{\alpha} \delta g^{\kappa \lambda}
$$

where,

$$
\delta n^{\alpha}=-\frac{1}{2} n^{\alpha} n_{\kappa} n_{\lambda} \delta g^{\kappa \lambda}+\delta g^{\alpha \beta} n_{\beta} .
$$

Using these relations, we obtain, after some simplification,

$$
\begin{aligned}
\delta I_{\left[\partial \mathrm{AdS}_{2} \rightarrow \infty\right]}-\delta I_{\mathrm{GH}}= & \frac{1}{16 \pi \widetilde{G}} \int_{\partial \mathrm{AdS}_{2}} \mathrm{~d} x \sqrt{\gamma}\left(\nabla_{\alpha}\left(\phi n_{\beta} \delta g^{\alpha \beta}\right)+\delta g^{\alpha \beta} \phi\left(\nabla_{\alpha} n_{\beta}\right)\right) \\
& +\frac{1}{16 \pi \widetilde{G}} \int_{\partial \mathrm{AdS}_{2}} \mathrm{~d} x \sqrt{\gamma}\left(\phi \nabla_{\kappa}\left(n^{\kappa} \gamma_{\alpha \beta} \delta g^{\alpha \beta}\right)-\nabla_{\kappa}\left(\phi n^{\kappa} g_{\alpha \beta} \delta g^{\alpha \beta}\right)\right) \\
& +\frac{1}{8 \pi \widetilde{G}} \int_{\partial \mathrm{AdS}_{2}} \mathrm{~d} x \delta(\sqrt{\gamma}) \phi K,
\end{aligned}
$$


where,

$$
\gamma_{\alpha \beta}=g_{\alpha \beta}-n_{\alpha} n_{\beta} .
$$

To proceed, we use a coordinate system, with $r, t$ coordinates, in which the metric of $\mathrm{AdS}_{2}$ is

$$
g_{t t}=\frac{1}{g_{r r}}=\frac{\left(r-r_{h}\right)^{2}}{L_{2}^{2}}, \quad g_{t r}=0,
$$

and the dilaton, eq. (2.35) becomes,

$$
\phi=\frac{r-r_{h}}{r_{h}}
$$

This gives on the constant $r$ hyper-surface on the $\mathrm{AdS}_{2}$ boundary, the unit normal one-form,

$$
n_{r}=\frac{L_{2}}{r-r_{h}}, \quad n_{t}=0 .
$$

In this coordinate system the metric components, eq. (A.12), change as follows, when the mass $M$, eq. (2.16) is changed by $\delta M$ to go to the non-extremal case:

$$
\delta g^{t t}=\frac{2 L_{2}^{4} G_{4} \delta M}{r_{h}\left(r-r_{h}\right)^{4}}, \quad \delta g^{r r}=-\frac{2 G_{4} \delta M}{r_{h}}, \quad \delta g^{t r}=0 .
$$

Using this explicit form, we find that the first two lines of (A.10) vanish and we are left with,

$$
\delta I_{\left[\partial \mathrm{AdS}_{2} \rightarrow \infty\right]}-\delta I_{\mathrm{GH}}=\frac{1}{8 \pi \widetilde{G}} \int_{\partial \mathrm{AdS}_{2}} \mathrm{~d} x \delta(\sqrt{\gamma}) \phi K .
$$

Since $K=1 / L_{2}$, we have,

$$
\delta I_{\left[\partial \mathrm{AdS}_{2} \rightarrow \infty\right]}=\delta I_{\mathrm{GH}}+\frac{1}{8 \pi \widetilde{G} L_{2}} \int_{\partial \mathrm{AdS}_{2}} \mathrm{~d} x \delta(\sqrt{\gamma}) \phi .
$$

We thus see that the change to leading order in $I_{\left[\partial \mathrm{AdS}_{2} \rightarrow \infty\right]}$ agrees with the change in the Gibbons-Hawking boundary term and the associated counter-term on the $\mathrm{AdS}_{2}$ boundary.

Before we end this appendix, let us make two comments. First, with reference to the discussion in 3.3, instead of starting with the action, eq. (A.1), consider the linearised action of the JT model, eq. (3.5) which is now evaluated in the region extending from the horizon to the boundary of $\mathrm{AdS}_{2}$. And let us take an alteration of the metric which can be regarded as a small perturbation in this region. Then steps which are very analogous to those above lead to a change given in eq. (3.44).

Second, consider the general two-dimensional theory of section 3.2. All the above arguments go through as before. The new ingredient in this case is the presence of the extra scalars $\Psi_{p}$. When we vary the far-horizon action, some surface terms are generated on the $\mathrm{AdS}_{2}$ boundary. The relevant term in the action (3.18) is,

$$
-\frac{v_{d-1}}{16 \pi G_{d+1}} \int \mathrm{d}^{2} x \sqrt{g} h^{p q}\left(\Phi, \Psi_{i}\right) g^{\alpha \beta} \nabla_{\alpha} \Psi_{p} \nabla_{\beta} \Psi_{q}
$$


A variation of this action yields,

$$
-\frac{v_{d-1}}{8 \pi G_{d+1}} \int_{\partial \mathrm{AdS}_{2}} \mathrm{~d} x \sqrt{\gamma} h^{p q} n^{\alpha} \partial_{\alpha} \Psi_{p} \delta \Psi_{q} .
$$

Unlike in the case of $\Phi$, no Dirichlet condition on $\Psi$ is imposed at the $\mathrm{AdS}_{2}$ boundary. Therefore, the above expression does not vanish, in general. However, it is easy to see that this does not contribute to the leading thermodynamic behaviour we are interested in. Recall from section 3.2 that $\Psi_{i}$ also shows the behaviour (3.21). In the asymptotic $\mathrm{AdS}_{2}$ region the effects of the mass deformation die out and this implies that $\delta \Psi_{i}$ will also go as $\mathcal{J}_{i}^{-1}$. The net contribution of this boundary term will therefore be suppressed at least as $\mathcal{J}_{i}^{-2}$. Since $\mathcal{J}$ and $\mathcal{J}_{i}$ are assumed to be of similar order, this contribution is suppressed in comparison to the leading thermodynamic behaviour which goes as $\mathcal{J}^{-1}$.

Let us comment on the behaviour of $\delta \Psi_{i}$ in the asymptotic $\mathrm{AdS}_{2}$ region in some more detail. Consider the mass deformed two-dimensional metric which can be written as,

$$
\mathrm{d} s^{2}=\left(r^{2}-\delta_{0}^{2}\right) \mathrm{d} t^{2}+\frac{\mathrm{d} r^{2}}{\left(r^{2}-\delta_{0}^{2}\right)} .
$$

Here we have relabelled to the coordinate $\left(r-r_{h}\right)$ used in section 2 as $r$ and $\delta_{0} \sim T$. A perturbation $\delta \Psi_{i}$ of mass $m$ which is time-independent satisfies the equation

$$
\partial_{r}\left[\left(r^{2}-\delta_{0}^{2}\right) \partial_{r} \delta \Psi_{i}\right]-m^{2} \delta \Psi_{i}=0 .
$$

In the asymptotic $\mathrm{AdS}_{2}$ region, $r^{2} \gg \delta_{0}^{2} \sim T^{2}$, eq. (A.21) becomes the same as in the undeformed case. Regularity at the horizon chooses one of the two solutions of eq. (A.21); the remaining overall coefficient which specifies this solution is fixed by matching with the behaviour in the asymptotic $\mathrm{AdS}_{2}$ region. This allows the solution to meet the boundary conditions at infinity. For the $m^{2}=2$ case, for instance, the behaviour in the asymptotic $\mathrm{AdS}_{2}$ region goes like $\sim r / \mathcal{J}_{i}$, and in fact,

$$
\delta \Psi_{i}=\frac{r}{L_{2}^{2} \mathcal{J}_{i}}
$$

solves eq. (A.21) identically. As the value of the dilaton in the asymptotic region changes due to the mass deformation, the boundary of the $\mathrm{AdS}_{2}$ region also changes, however $\delta \Psi_{i}$ will continue to be of order $1 / \mathcal{J}_{i}$.

\section{B Electrically charged near-extremal black holes in $(d+1)$ dimensions}

The electrically charged RN case in general $d+1$ dimensions has been studied earlier, [102, 158]. Here we review how the free energy for this case agrees with the JT model.

We consider Euclidean Einstein-Maxwell action in asymptotically $\mathrm{AdS}_{d+1}$ spacetime, appropriate for the canonical ensemble,

$$
\begin{aligned}
I= & -\frac{1}{16 \pi G_{d+1}} \int \mathrm{d}^{d+1} x \sqrt{g}\left(R-2 \Lambda_{d+1}+F_{\mu \nu} F^{\mu \nu}\right) \\
& -\frac{1}{8 \pi G_{d+1}} \int \mathrm{d}^{d} x \sqrt{\gamma}\left(K-2 n_{\mu} F^{\mu \nu} A_{\nu}\right) .
\end{aligned}
$$


The electrically charged Reissner-Nordström solution is given by,

$$
\mathrm{d} s^{2}=f(r) \mathrm{d} t^{2}+\frac{\mathrm{d} r^{2}}{f(r)}+r^{2} \mathrm{~d} \Omega_{d-1}^{2}
$$

where,

$$
\begin{aligned}
f(r) & =\left(1-\frac{2 m}{r^{d-2}}+\frac{q^{2}}{r^{2 d-4}}+\frac{r^{2}}{L^{2}}\right), \\
L & =\sqrt{-\frac{d(d-1)}{2 \Lambda_{d+1}}}, \\
F_{r t} & =\sqrt{\frac{(d-2)(d-1)}{2}} \frac{q}{r^{d-1}} .
\end{aligned}
$$

The physical mass is given by,

$$
M=M_{\text {Casimir }}+\frac{(d-1) v_{d-1} m}{8 \pi G_{d+1}} .
$$

Here we have defined

$$
v_{d} \equiv \operatorname{Vol}\left(S^{d}\right)=\frac{2 \pi^{\frac{d+1}{2}}}{\Gamma\left(\frac{d+1}{2}\right)} .
$$

We have, in general, a non-zero $M_{\text {Casimir }}$ when $d$ is even.

In the extremal case $f(r)$, eq. (B.3) has a double zero at the horizon, $r=r_{h}$. Increasing the mass by $\delta M$ keeping the charge parameter $q$ fixed, we find the near-horizon metric to be,

$$
\mathrm{d} s^{2}=\frac{\left(r-r_{h}\right)^{2}-\delta_{0}^{2}}{L_{2}^{2}} \mathrm{~d} t^{2}+\frac{L_{2}^{2}}{\left(r-r_{h}\right)^{2}-\delta_{0}^{2}} \mathrm{~d} r^{2}+r^{2} \mathrm{~d} \Omega_{d-1}^{2},
$$

where,

$$
\begin{aligned}
\frac{1}{L_{2}^{2}} & =\frac{d(d-1)}{L^{2}}+\frac{(d-2)^{2}}{r_{h}^{2}} \\
\delta_{0}^{2} & =\frac{16 \pi G_{d+1} L_{2}^{2} \delta M}{(d-1) v_{d-1} r_{h}^{d-2}}
\end{aligned}
$$

It is easy to see that the temperature is given by

$$
T=\frac{\delta_{0}}{2 \pi L_{2}^{2}},
$$

and the entropy above extremality is given by

$$
\Delta S=\frac{\pi(d-1) v_{d-1} L_{2}^{2} r_{h}^{d-2}}{2 G_{d+1}} T .
$$

This leads to a free energy (using the usual thermodynamic relation),

$$
\Delta F=-\frac{\pi(d-1) v_{d-1} L_{2}^{2} r_{h}^{d-2}}{4 G_{d+1}} T^{2}
$$


Next let us compare this with the result in the JT model obtained in a manner analogous to what was described in section 2 for the four-dimensional case. Dimensionally reducing the action (B.1) with the ansatz,

$$
\mathrm{d} s^{2}=\left(\frac{\Phi_{0}}{\Phi}\right)^{d-2} g_{\alpha \beta} \mathrm{d} x^{\alpha} \mathrm{d} x^{\beta}+\Phi^{2} \mathrm{~d} \Omega_{d-1}^{2} .
$$

gives the two-dimensional action,

$$
\begin{gathered}
I=-\frac{v_{d-1}}{16 \pi G_{d+1}} \int \mathrm{d}^{2} x \sqrt{g}\left(\Phi^{d-1} R+\frac{(d-1)(d-2) \Phi_{0}^{d-2}}{\Phi}\right. \\
\left.+\frac{d(d-1)}{L^{2}} \Phi_{0}^{d-2} \Phi+\frac{\Phi^{2 d-3}}{\Phi_{0}^{d-2}} F_{\alpha \beta} F^{\alpha \beta}\right) \\
-\frac{v_{d-1}}{8 \pi G_{d+1}} \int \mathrm{d} x \sqrt{\gamma}\left(\Phi^{d-1} K-\frac{2 \Phi^{2 d-3}}{\Phi_{0}^{d-2}} n_{\alpha} F^{\alpha \beta} A_{\beta}\right) .
\end{gathered}
$$

Here

$$
\Phi=r
$$

and $\Phi_{0}=r_{h}$ is the attractor value for $\Phi$. Expanding about the attractor value we see that,

$$
\Phi=\Phi_{0}\left(1+\frac{r-r_{h}}{r_{h}}\right)=\Phi_{0}\left(1+\frac{1}{\mathcal{J} z}\right) .
$$

where $\mathcal{J}$, which determines the scale of conformal symmetry breaking in the near-horizon region eq. (2.5), is

$$
\mathcal{J}=\frac{r_{h}}{L_{2}^{2}}
$$

We get from the equations of motion,

$$
F_{\alpha \beta}=\frac{\Phi_{0}^{d-2}}{\Phi^{2 d-3}} Q \sqrt{g} \epsilon_{\alpha \beta},
$$

where,

$$
Q^{2}=\frac{1}{2}(d-1)(d-2) q_{0}^{2}
$$

with $q_{0}$ referring to the charge parameter at extremality.

Following the procedure outlined in section 3 and expanding about the attractor value of $\Phi$, we readily obtain the bulk action,

$$
I=-\frac{v_{d-1}(d-1) r_{h}^{d-1}}{16 \pi G_{d+1}} \int \mathrm{d}^{2} x \sqrt{g} \phi\left(R+\frac{2}{L_{2}^{2}}\right) .
$$

This agrees with the JT model bulk action in eq. (2.1) and allows us to identify,

$$
\widetilde{G}=\frac{G_{d+1}}{(d-1) v_{d-1} r_{h}^{d-1}} .
$$

From eq. (2.14), eq. (B.18) and eq. (B.22) we see that the free energy of this JT model also agrees with eq. (B.13) above. 


\section{Rotating black holes in five dimensions in asymptotically Anti-de Sit- ter spacetime}

In this appendix, we give further details on the five-dimensional rotating black hole, discussed in section 4. The five-dimensional action is given by eq. (4.1) in which the fivedimensional cosmological constant is given by,

$$
\Lambda_{5}=-\frac{6}{L^{2}} .
$$

The five-dimensional rotating black hole solution [86] is given by eq. (4.2), where,

$$
\begin{aligned}
\Xi & =1-\frac{a^{2}}{L^{2}}, \\
\Delta & =1+\frac{r^{2}}{L^{2}}-\frac{2 m r^{2}}{\left(a^{2}+r^{2}\right)^{2}}, \\
\exp \left(-U_{1}\right) & =\frac{a^{2}+r^{2}}{4 \Xi}, \\
\exp \left(-U_{2}\right) & =\frac{m a^{2}}{2 \Xi^{2}\left(a^{2}+r^{2}\right)}+\frac{a^{2}+r^{2}}{4 \Xi}, \\
A & =\cos \theta \mathrm{d} \varphi-\mathrm{i} \frac{a}{2 \Xi}\left(\frac{a^{2}+r^{2}}{L^{2}}-\frac{2 m}{a^{2}+r^{2}}\right) \exp \left(U_{2}\right) \mathrm{d} t .
\end{aligned}
$$

The physical mass $M$ and the angular momentum are given by, respectively,

$$
\begin{aligned}
M & =\frac{3 \pi L^{2}}{32 G_{5}}+\frac{3 \pi\left(1+\frac{a^{2}}{3 L^{2}}\right)}{4 G_{5} \Xi^{3}} m, \\
J & =\frac{\pi m a}{G_{5} \Xi^{3}} .
\end{aligned}
$$

Here, the first term in (C.7) corresponds to the Casimir energy for this spacetime. The entropy and the temperature are given respectively by,

$$
\begin{aligned}
& S=\frac{\pi^{2}\left(r_{+}^{2}+a^{2}\right)^{2}}{2 G_{5} r_{+} \Xi^{2}}, \\
& T=\frac{r_{+}^{2}-a^{2}+\frac{2 r_{+}^{4}}{L^{2}}}{2 \pi r_{+}\left(r_{+}^{2}+a^{2}\right)},
\end{aligned}
$$

where $r_{+}$denotes the location of the outer horizon. Note that the above formulae make it obvious that the absolute value of the parameter $a$ cannot be arbitrarily large. It can, at best, approach $L$ from below.

The angular velocity which is thermodynamic conjugate of the angular momentum [159] is given by,

$$
\Omega_{H}^{\infty}=a \frac{\left(1+\frac{r_{+}^{2}}{L^{2}}\right)}{r_{+}^{2}+a^{2}} .
$$

At extremality, we have the relations,

$$
\begin{aligned}
& m_{0}=2 r_{h}^{2}\left(1+\frac{r_{h}^{2}}{L^{2}}\right)^{3}, \\
& a_{0}=r_{h}\left(1+\frac{2 r_{h}^{2}}{L^{2}}\right)^{1 / 2}
\end{aligned}
$$


The fixed angular momentum condition gives us,

$$
\begin{aligned}
& m=m_{0}+\delta m, \\
& a=a_{0}-\frac{\left(1-\frac{2 r_{h}^{2}}{L^{2}}\right)\left(1+\frac{2 r_{h}^{2}}{L^{2}}\right)^{1 / 2}}{\left(1+\frac{r_{h}^{2}}{L^{2}}\right)^{2}\left(1+\frac{5 r_{h}^{2}}{L^{2}}+\frac{10 r_{h}^{4}}{L^{4}}\right)} \frac{\delta m}{2 r_{h}} .
\end{aligned}
$$

The horizon is shifted to,

$$
r_{+}=r_{h}+\delta_{0}+\mathcal{O}\left(\delta_{0}^{2}\right)
$$

where

$$
\delta_{0}^{2}=\frac{3 \delta m}{2} \frac{\left(1+\frac{2 r_{h}^{2}}{3 L^{2}}\right)}{\left(1+\frac{4 r_{h}^{2}}{L^{2}}\right)\left(1+\frac{5 r_{h}^{2}}{L^{2}}+\frac{10 r_{h}^{4}}{L^{4}}\right)} .
$$

The extra mass above extremality and the temperature are related as,

$$
\delta M=\frac{2 \pi^{3} r_{h}^{2} L_{2}^{2}}{G_{5}} \frac{\left(1-\frac{r_{h}^{2}}{L^{2}}\right)}{\left(1+\frac{r_{h}^{2}}{L^{2}}\right)\left(1-\frac{2 r_{h}^{2}}{L^{2}}\right)^{2}} T^{2} .
$$

The $L_{2}$ used here is defined in eq. (4.5). At small temperature, the excess entropy is found to be,

$$
\Delta S=\frac{12 \pi^{3} r_{h}^{3}}{G_{5}\left(1-\frac{2 r_{h}^{2}}{L^{2}}\right)^{2}} \frac{T}{\mathcal{J}},
$$

From which we easily obtain the form of free energy as in eq. (4.6), where $\widetilde{G}, \mathcal{J}$ are defined in eqs. (4.7), (4.8).

A criterion for superradiance instability to occur [144] is given by,

$$
\Omega_{H}^{\infty}>\frac{1}{L}
$$

Since $a$ can approach $L$ only from below, it it easy to see that this criterion is equivalent to demanding that,

$$
r_{+}^{2}<a L
$$

In the purely rotating five-dimensional near-extremal case, one finds that this criterion is always met and hence an instability could exist [144].

\section{C.1 Dimensional reduction to two dimensions}

We now perform the dimensional reduction of the action (4.1) - first with the metric ansatz (4.9) to obtain the four-dimensional action (4.10), then with the metric and gauge field ansatz (4.11), (4.13). The resulting two-dimensional action becomes, after we have added a gauge field boundary term for the canonical ensemble,

$$
\begin{aligned}
I= & -\frac{\pi}{8 G_{5}} \int \mathrm{d}^{2} x \sqrt{g}\left(\Phi^{3} R+\frac{16}{\Phi^{3 / 2}}-\frac{3 \Phi^{3}}{2 \Sigma^{2}}(\nabla \Sigma)^{2}-\frac{2 \Phi^{3 / 2}}{\Sigma} \Lambda_{5}-\frac{32 \Sigma^{3}}{\Phi^{9 / 2}}+\frac{\Phi^{9 / 2} \Sigma^{3}}{4} F_{\alpha \beta} F^{\alpha \beta}\right) \\
& -\frac{\pi}{4 G_{5}} \int \mathrm{d} x \sqrt{\gamma}\left(\Phi^{3} K-\frac{\Phi^{9 / 2} \Sigma^{3}}{2} n_{\alpha} F^{\alpha \beta} A_{\beta}\right) .
\end{aligned}
$$


The equation of motion for the gauge field gives,

$$
F_{\alpha \beta}=\frac{Q}{\Phi^{9 / 2} \Sigma^{3}} \sqrt{g} \epsilon_{\alpha \beta},
$$

where the charge $Q$ is given by,

$$
Q^{2}=32 \Sigma_{0}^{3} \Phi_{0}^{3}\left(1-\frac{\Phi_{0}^{3}}{8 \Sigma_{0}} \Lambda_{5}-\frac{2 \Sigma_{0}^{3}}{\Phi_{0}^{3}}\right)=\left(\frac{4 G_{5}}{\pi} J_{0}\right)^{2} .
$$

Here, $J_{0}$ is the extremal value of the angular momentum (C.8) and $\Phi_{0}, \Sigma_{0}$ are the attractor values of the corresponding scalars. Comparing with the attractor solution (4.3), we readily find that

$$
\Sigma_{0}=\frac{r_{h}}{\left(1-\frac{2 r_{h}^{2}}{L^{2}}\right)},
$$

with the value of $\Phi_{0}$ given by eq. (4.15).

Now, following the general procedure discussed in section 3 , and linearising about the attractor solutions, $\Phi=\Phi_{0}(1+\phi)$ and $\Sigma=\Sigma_{0}(1+\sigma)$, we have the bulk action in the JT form,

$$
I=-\frac{3 \pi r_{h}^{3}}{2 G_{5}\left(1-\frac{2 r_{h}^{2}}{L^{2}}\right)^{2}} \int \mathrm{d}^{2} x \sqrt{g} \phi\left(R+\frac{2 \lambda}{L_{2}^{2}}\right),
$$

where,

$$
\lambda=\frac{1}{r_{h}^{5 / 2}}\left(1-\frac{2 r_{h}^{2}}{L^{2}}\right)^{2}\left(1+\frac{r_{h}^{2}}{L^{2}}\right) .
$$

Now, the constant Weyl rescaling,

$$
g_{\alpha \beta} \rightarrow \frac{1}{\lambda} g_{\alpha \beta}
$$

brings the action to the form (2.1) with $\widetilde{G}$ given by (4.7). It is worth pointing out that since we are expanding about the attractor eq. (C.25), eq. (4.15), there is no term in eq. (C.26) which is linear in $\sigma$.

\section{Rotating dyonic black holes in four dimensions in asymptotically Anti- de Sitter spacetime}

In this appendix, we elaborate on the four-dimensional set-up discussed in section 5 . The action, metric, gauge field and the various parameters are described in eqs. (5.1)-(5.14).

The expressions for entropy and temperature are given respectively by,

$$
\begin{aligned}
& S=\frac{\pi}{\Xi G_{4}}\left(r_{+}^{2}+a^{2}\right), \\
& T=\frac{\left(r_{+}^{2}-a^{2}-q_{e}^{2}-q_{m}^{2}\right)+\frac{r_{+}^{2}}{L^{2}}\left(3 r_{+}^{2}+a^{2}\right)}{4 \pi r_{+}\left(r_{+}^{2}+a^{2}\right)} .
\end{aligned}
$$


Here $r_{+}$is the location of the outer horizon, $\Delta_{r}\left(r_{+}\right)=0$. The angular velocity at the horizon is given by,

$$
\Omega_{H} \equiv \omega\left(r_{+}\right)=\frac{a \Xi}{r_{+}^{2}+a^{2}} .
$$

As in the five-dimensional case, the angular velocity which is thermodynamically conjugate to the angular momentum [159] is given by,

$$
\Omega_{H}^{\infty}=a \frac{\left(1+\frac{r_{+}^{2}}{L^{2}}\right)}{r_{+}^{2}+a^{2}} .
$$

Let us now examine the black hole around extremality. Denoting all extremal quantities with a subscript 0 and defining

$$
q_{0}^{2} \equiv q_{e 0}^{2}+q_{m 0}^{2},
$$

the mass parameter $m_{0}$ and and the angular momentum parameter $a_{0}$ are parametrically related as,

$$
\begin{aligned}
& m_{0}=r_{h} \frac{\left(1+\frac{2 r_{h}^{2}-q_{0}^{2}}{L^{2}}+\frac{r_{h}^{4}}{L^{2}}\right)}{\left(1-\frac{r_{h}^{2}}{L^{2}}\right)}, \\
& a_{0}=r_{h}\left(\frac{1+\frac{3 r_{h}^{2}}{L^{2}}-\frac{q_{0}^{2}}{r_{h}^{2}}}{1-\frac{r_{h}^{2}}{L^{2}}}\right)^{1 / 2},
\end{aligned}
$$

where $r_{h}$ is the radial location of the extremal horizon.

For a concise presentation of the later results, it is useful to express the mass and charge parameters as functions of the extremal radius and the angular momentum parameter. So we have,

$$
\begin{aligned}
m_{0} & =r_{h}\left(1+\frac{a_{0}^{2}+2 r_{h}^{2}}{L^{2}}\right) \\
q_{0} & =\sqrt{r_{h}^{2}\left(1+\frac{3 r_{h}^{2}}{L^{2}}\right)-a_{0}^{2}\left(1-\frac{r_{h}^{2}}{L^{2}}\right)} .
\end{aligned}
$$

We are interested in making the black hole slightly non-extremal by adding a small amount of mass, but keeping the angular momentum and the electric and magnetic charges unchanged. This necessitates that we take,

$$
\begin{aligned}
m & =m_{0}+\delta m \\
a & =a_{0}\left(1-\frac{\Xi_{0}}{\left(1+\frac{3 a_{0}^{2}}{L^{2}}\right)} \frac{\delta m}{m_{0}}\right), \\
q_{e} & =q_{e 0}\left(1+\frac{2 a_{0}^{2}}{L^{2}+3 a_{0}^{2}} \frac{\delta m}{m_{0}}\right), \\
q_{m} & =q_{m 0}\left(1+\frac{2 a_{0}^{2}}{L^{2}+3 a_{0}^{2}} \frac{\delta m}{m_{0}}\right),
\end{aligned}
$$


Then, the outer horizon is shifted to,

$$
r_{+}=r_{h}+\delta_{0}+\mathcal{O}\left(\delta_{0}^{2}\right)
$$

where

$$
\delta_{0}^{2}=\frac{2\left(r_{h}^{2}+a_{0}^{2}\right)}{r_{h}\left(1+\frac{3 a_{0}^{2}}{L^{2}}\right)\left(1+\frac{6 r_{h}^{2}+a_{0}^{2}}{L^{2}}\right)} \delta m .
$$

The mass above extremality and the temperature are related as,

$$
\delta M=\frac{2 \pi^{2} r_{h}\left(r_{h}^{2}+a_{0}^{2}\right)}{G_{4} \Xi_{0}\left(1+\frac{6 r_{h}^{2}+a_{0}^{2}}{L^{2}}\right)} T^{2} .
$$

The entropy above extremality then goes as,

$$
\Delta S=\frac{4 \pi^{2} r_{h}\left(r_{h}^{2}+a_{0}^{2}\right)}{G_{4} \Xi_{0}\left(1+\frac{6 r_{h}^{2}+a_{0}^{2}}{L^{2}}\right)} T .
$$

Here,

$$
\Xi_{0}=1-\frac{a_{0}^{2}}{L^{2}}
$$

We now look at the near-horizon limit of the metric. We make the scalings as in [121, 137], in a slightly more generalised form to accommodate deviations from extremality,

$$
\begin{aligned}
t & \rightarrow \lambda^{-1} t, \\
r-r_{h} & \rightarrow \lambda\left(r-r_{h}\right), \\
\varphi & \rightarrow \varphi-\mathrm{i} \lambda^{-1} \Omega_{H} t, \\
m & =m_{0}+\lambda^{2} \delta m, \\
a & =a_{0}\left(1-\lambda^{2} \frac{\Xi_{0}}{\left(1+\frac{3 a_{0}^{2}}{L^{2}}\right)} \frac{\delta m}{m_{0}}\right), \\
q_{(e, m)} & =q_{(e 0, m 0)}\left(1+\lambda^{2} \frac{2 a_{0}^{2}}{L^{2}+3 a_{0}^{2}} \frac{\delta m}{m_{0}}\right) .
\end{aligned}
$$

Putting this into eq. (5.2) and taking the limit $\lambda \rightarrow 0$ gives us the attractor solution,

$$
\begin{aligned}
\mathrm{d} s^{2}= & \frac{\rho_{0}^{2}}{\left(r_{h}^{2}+a_{0}^{2}\right)}\left(\frac{\left(r-r_{h}\right)^{2}-\delta_{0}^{2}}{L_{2}^{2}} \mathrm{~d} t^{2}+\frac{L_{2}^{2}}{\left(r-r_{h}\right)^{2}-\delta_{0}^{2}} \mathrm{~d} r^{2}\right)+\frac{\rho_{0}^{2} \Xi_{0}}{\left(r_{h}^{2}+a_{0}^{2}\right) \Delta_{\theta 0}} \Phi_{0}^{2} \mathrm{~d} \theta^{2} \\
& +\frac{\Delta_{\theta 0}\left(r_{h}^{2}+a_{0}^{2}\right)}{\Xi_{0} \rho_{0}^{2}} \Phi_{0}^{2} \sin ^{2} \theta\left(\mathrm{d} \varphi-\mathrm{i} \frac{2 r_{h} a_{0} \Xi_{0}}{\left(r_{h}^{2}+a_{0}^{2}\right)^{2}}\left(r-r_{h}\right) \mathrm{d} t\right)^{2},
\end{aligned}
$$

where,

$$
\begin{aligned}
L_{2}^{2} & =\frac{r_{h}^{2}+a_{0}^{2}}{\left(1+\frac{6 r_{h}^{2}+a_{0}^{2}}{L^{2}}\right)}, \\
\rho_{0}^{2} & =r_{h}^{2}+a_{0}^{2} \cos ^{2} \theta,
\end{aligned}
$$




$$
\begin{aligned}
\Phi_{0}^{2} & =\frac{r_{h}^{2}+a_{0}^{2}}{\Xi_{0}}, \\
\Delta_{\theta 0} & =1-\frac{a_{0}^{2}}{L^{2}} \cos ^{2} \theta .
\end{aligned}
$$

The near-horizon gauge field takes the form, after removing a part going as $k \mathrm{~d} t$ by a gauge transformation,

$$
\begin{aligned}
\hat{A}_{\mu} \mathrm{d} x^{\mu}= & \frac{q_{e 0}}{\Xi_{0} \rho_{0}^{2}}\left(-\mathrm{i} \frac{\left(r_{h}^{2}-a_{0}^{2} \cos ^{2} \theta\right) \Xi_{0}}{r_{h}^{2}+a_{0}^{2}}\left(r-r_{h}\right) \mathrm{d} t+a_{0} r_{h} \sin ^{2} \theta \mathrm{d} \varphi\right) \\
& +\frac{q_{m 0} \cos \theta}{\Xi_{0} \rho_{0}^{2}}\left(-\mathrm{i} \frac{2 a_{0} r_{h} \Xi_{0}}{r_{h}^{2}+a_{0}^{2}}\left(r-r_{h}\right) \mathrm{d} t+\left(r_{h}^{2}+a_{0}^{2}\right) \mathrm{d} \varphi\right) .
\end{aligned}
$$

Higher order corrections to the attractor metric and the gauge field can be obtained by retaining terms of higher orders in $\lambda$. Even the first order corrections to the metric are rather complicated.

\section{D.1 Dimensional reduction to two dimensions}

Next, we reduce the action (5.1) with metric and gauge field ansatz to be of the form (5.23) and (5.24), respectively. Note that we take $\Phi, A_{\alpha}, \mathcal{A}_{\alpha}$ to be only dependent on coordinates $x^{\alpha}$.

The resulting two-dimensional action then becomes, after including appropriate boundary terms for the canonical ensemble,

$$
\begin{aligned}
I= & -\frac{1}{4 G_{4}} \int \mathrm{d}^{2} x \sqrt{g}\left(R \Phi^{2}+c_{1} \frac{\Phi_{0}}{\Phi}+c_{2} \Phi_{0} \Phi-c_{3} \frac{\Phi_{0}}{\Phi^{3}}+\frac{\Phi^{3}}{\Phi_{0}}\left(c_{4} \Phi^{2}+c_{5}\right) F_{\alpha \beta} F^{\alpha \beta}\right) \\
& -\frac{1}{4 G_{4}} \int \mathrm{d}^{2} x \sqrt{g} \frac{\Phi^{3}}{\Phi_{0}}\left(c_{6} \mathcal{F}_{\alpha \beta} \mathcal{F}^{\alpha \beta}+c_{7} \mathcal{F}_{\alpha \beta} F^{\alpha \beta}\right)-\frac{1}{2 G_{4}} \int \mathrm{d} x \sqrt{\gamma} \Phi^{2} K \\
& +\frac{1}{G_{4}} \int \mathrm{d} x \sqrt{\gamma} \frac{\Phi^{3}}{\Phi_{0}} n_{\alpha}\left(\left(c_{4} \Phi^{2}+c_{5}\right) F^{\alpha \beta} A_{\beta}+c_{6} \mathcal{F}^{\alpha \beta} \mathcal{A}_{\beta}+\frac{c_{7}}{2}\left(F^{\alpha \beta} \mathcal{A}_{\beta}+\mathcal{F}^{\alpha \beta} A_{\beta}\right)\right) .
\end{aligned}
$$

where,

$$
\begin{aligned}
& c_{1}=\frac{1}{\Xi_{0}}\left(1-\frac{r_{h}^{2}}{L^{2}}\left(3+2 \chi^{2}\right)\right)+\frac{1}{\Xi_{0} \chi}\left(\left(1-\chi^{2}\right)+\frac{r_{h}^{2}}{L^{2}}\left(3+\chi^{2}\right)\right) \tan ^{-1} \chi, \\
& c_{2}=\frac{2}{L^{2}} \frac{3+\chi^{2}}{1+\chi^{2}}, \\
& c_{3}=\frac{1}{\Xi_{0}^{2} \chi\left(1+\chi^{2}\right)}\left(q_{0}^{2}\left(1+\chi^{2}\right)^{2} \tan ^{-1} \chi-\left(q_{e 0}^{2}-q_{m 0}^{2}\right) \chi\left(1-\chi^{2}\right)\right), \\
& c_{4}=\frac{\left(1+\chi^{2}\right)^{2}}{8 \chi^{3} \Xi_{0}}\left[\left(1+\frac{3 r_{h}^{2}}{L^{2}}\right) \chi-\left(1-\chi^{2}+\frac{r_{h}^{2}}{L^{2}}\left(3+\chi^{2}\right)\right) \tan ^{-1} \chi\right], \\
& c_{5}=\frac{\left(1+\chi^{2}\right)}{8 \chi^{3} \Xi_{0}^{2}}\left[\left(q_{m 0}^{2}\left(1+\chi^{2}\right)^{2}+q_{e 0}^{2}\left(3-2 \chi^{2}+3 \chi^{4}\right)\right) \tan ^{-1} \chi-\left(3 q_{e 0}^{2}+q_{m 0}^{2}\right) \chi\left(1-\chi^{2}\right)\right], \\
& c_{6}=\frac{\left(1+\chi^{2}\right)}{\chi} \tan ^{-1} \chi, \\
& c_{7}=\frac{q_{e 0}\left(1+\chi^{2}\right)\left[\chi-\left(1-\chi^{2}\right) \tan ^{-1} \chi\right]}{\chi^{2} \Xi_{0}} .
\end{aligned}
$$


Here, we have defined,

$$
\chi=\frac{a_{0}}{r_{h}},
$$

and have used the standard notation for field strength,

$$
F_{\alpha \beta}=\partial_{\alpha} A_{\beta}-\partial_{\beta} A_{\alpha}, \quad \mathcal{F}_{\alpha \beta}=\partial_{\alpha} \mathcal{A}_{\beta}-\partial_{\beta} \mathcal{A}_{\alpha} .
$$

In the two-dimensional theory, we have two U(1) gauge fields. The equations of motion for $A_{\alpha}$ and $\mathcal{A}_{\alpha}$, give respectively,

$$
\begin{aligned}
\frac{\Phi^{3}}{\Phi_{0}}\left(c_{4} \Phi^{2}+c_{5}\right) F_{\alpha \beta}+c_{7} \frac{\Phi^{3}}{2 \Phi_{0}} \mathcal{F}_{\alpha \beta} & =Q \sqrt{g} \epsilon_{\alpha \beta}, \\
\frac{c_{6} \Phi^{3}}{\Phi_{0}} \mathcal{F}_{\alpha \beta}+c_{7} \frac{\Phi^{3}}{2 \Phi_{0}} F_{\alpha \beta} & =\mathcal{Q} \sqrt{g} \epsilon_{\alpha \beta} .
\end{aligned}
$$

It is easy to see from the attractor values (5.20), (5.21) that these charges are nothing but the extremal values of the angular momentum and the electric charge:

$$
Q=G_{4} J_{0}, \quad \mathcal{Q}=Q_{e 0},
$$

as one would expect. As suggested by the notation, the attractor value of the field $\Phi$ is given by $\Phi_{0}$ and the attractor geometry is an $\mathrm{AdS}_{2}$ with,

$$
R=-\frac{2}{L_{2}^{2}}
$$

Following the general procedure discussed in section 3.3, and expanding $\Phi$ about its attractor value $\Phi_{0}$, eq. (2.30), eq. (D.23), we obtain the bulk action of the JT model,

$$
I=-\frac{\left(r_{h}^{2}+a_{0}^{2}\right)}{2 G_{4}\left(1-\frac{a_{0}^{2}}{L^{2}}\right)} \int \mathrm{d}^{2} x \sqrt{g} \phi\left(R+\frac{2}{L_{2}^{2}}\right)
$$

From the above, we identify the two-dimensional Newton's constant in the JT model to be given by eq. (5.16).

From the full metric (5.2), we get the field $\Phi$ to be,

$$
\Phi^{2}=\frac{1}{\Xi} \sqrt{\frac{\Sigma}{\Delta_{\theta}}} .
$$

At the horizon in the extremal case, $\Delta_{r}$ which appears in $\Sigma$ has a second order zero. As a result we can set $\Delta_{r}$ to vanish in obtaining the linear variation of $\Phi$ away from the horizon. This gives,

$$
\Phi=\Phi_{0}\left(1+\frac{r-r_{h}}{L_{2}^{2} \mathcal{J}}\right)=\Phi_{0}\left(1+\frac{1}{\mathcal{J} z}\right),
$$

where $\mathcal{J}$ is as defined in eq. (5.17).

By taking certain limits of the parameters, one can easily obtain different special cases:

- The asymptotically flat spacetime limit is obtained by taking $L \rightarrow \infty$ 
- The purely rotating limit is obtained by taking $q_{e 0}, q_{m 0} \rightarrow 0$ and $\mathcal{A}_{\alpha} \rightarrow 0$.

- The purely magnetic limit (with rotation) is obtained by taking $q_{e 0} \rightarrow 0$ and $\mathcal{A}_{\alpha} \rightarrow 0$.

- The purely electric limit (with rotation) is obtained by taking $q_{m 0} \rightarrow 0$.

- The non-rotating limit is obtained by taking $a_{0} \rightarrow 0$ and $A_{\alpha} \rightarrow 0$.

Open Access. This article is distributed under the terms of the Creative Commons Attribution License (CC-BY 4.0), which permits any use, distribution and reproduction in any medium, provided the original author(s) and source are credited.

\section{References}

[1] S. Sachdev and J. Ye, Gapless spin fluid ground state in a random, quantum Heisenberg magnet, Phys. Rev. Lett. 70 (1993) 3339 [cond-mat/9212030] [INSPIRE].

[2] A. Kitaev, A simple model of quantum holography, talks given at KITP, April 7 and May 27 (2015).

[3] J. Maldacena, D. Stanford and Z. Yang, Conformal symmetry and its breaking in two dimensional Nearly Anti-de-Sitter space, PTEP 2016 (2016) 12C104 [arXiv:1606.01857] [INSPIRE].

[4] C. Teitelboim, Gravitation and Hamiltonian structure in two space-time dimensions, Phys. Lett. B 126 (1983) 41.

[5] R. Jackiw, Lower dimensional gravity, Nucl. Phys. B 252 (1985) 343 [InSPIRE].

[6] A. Almheiri and J. Polchinski, Models of AdS 2 backreaction and holography, JHEP 11 (2015) 014 [arXiv: 1402.6334] [INSPIRE].

[7] P. Nayak et al., On the dynamics of near-extremal black holes, JHEP 09 (2018) 048 [arXiv: 1802.09547] [INSPIRE].

[8] U. Moitra, S.P. Trivedi and V. Vishal, Extremal and near-extremal black holes and near-CFT $T_{1}$, JHEP 07 (2019) 055 [arXiv:1808.08239] [INSPIRE].

[9] T. Muta and S.D. Odintsov, Two-dimensional higher derivative quantum gravity with constant curvature constraint, Prog. Theor. Phys. 90 (1993) 247 [INSPIRE].

[10] J.P.S. Lemos and P.M. Sa, Nonsingular constant curvature two-dimensional black hole, Mod. Phys. Lett. A 9 (1994) 771 [gr-qc/9309023] [INSPIRE].

[11] J.P.S. Lemos, Thermodynamics of the two-dimensional black hole in the Teitelboim-Jackiw theory, Phys. Rev. D 54 (1996) 6206 [gr-qc/9608016] [InSPIRE].

[12] J. Polchinski and V. Rosenhaus, The spectrum in the Sachdev-Ye-Kitaev model, JHEP 04 (2016) 001 [arXiv: 1601.06768] [INSPIRE].

[13] A. Jevicki, K. Suzuki and J. Yoon, Bi-local holography in the SYK model, JHEP 07 (2016) 007 [arXiv: 1603.06246] [INSPIRE].

[14] J. Maldacena and D. Stanford, Remarks on the Sachdev-Ye-Kitaev model, Phys. Rev. D 94 (2016) 106002 [arXiv : 1604.07818] [inSPIRE].

[15] K. Jensen, Chaos in AdS 2 holography, Phys. Rev. Lett. 117 (2016) 111601 [arXiv: 1605. 06098] [INSPIRE]. 
[16] I. Danshita, M. Hanada and M. Tezuka, Creating and probing the Sachdev-Ye-Kitaev model with ultracold gases: Towards experimental studies of quantum gravity, PTEP 2017 (2017) 083I01 [arXiv: 1606 .02454] [INSPIRE].

[17] J. Engelsöy, T.G. Mertens and H. Verlinde, An investigation of $A d S_{2}$ backreaction and holography, JHEP 07 (2016) 139 [arXiv: 1606.03438] [INSPIRE].

[18] A. Almheiri and B. Kang, Conformal symmetry breaking and thermodynamics of near-extremal black holes, JHEP 10 (2016) 052 [arXiv: 1606. 04108] [INSPIRE].

[19] D. Bagrets, A. Altland and A. Kamenev, Sachdev-Ye-Kitaev model as Liouville quantum mechanics, Nucl. Phys. B 911 (2016) 191 [arXiv:1607.00694] [INSPIRE].

[20] M. Cvetič and I. Papadimitriou, AdS $S_{2}$ holographic dictionary, JHEP 12 (2016) 008 [Erratum ibid. 01 (2017) 120] [arXiv: 1608.07018] [INSPIRE].

[21] A. Jevicki and K. Suzuki, Bi-local holography in the SYK model: perturbations, JHEP 11 (2016) 046 [arXiv: 1608.07567] [INSPIRE].

[22] Y. Gu, X.-L. Qi and D. Stanford, Local criticality, diffusion and chaos in generalized Sachdev-Ye-Kitaev models, JHEP 05 (2017) 125 [arXiv: 1609.07832] [INSPIRE].

[23] D.J. Gross and V. Rosenhaus, A generalization of Sachdev-Ye-Kitaev, JHEP 02 (2017) 093 [arXiv: 1610.01569] [INSPIRE].

[24] M. Berkooz, P. Narayan, M. Rozali and J. Simón, Higher dimensional generalizations of the SYK model, JHEP 01 (2017) 138 [arXiv:1610.02422] [INSPIRE].

[25] A.M. García-García and J.J.M. Verbaarschot, Spectral and thermodynamic properties of the Sachdev-Ye-Kitaev model, Phys. Rev. D 94 (2016) 126010 [arXiv:1610.03816] [InSPIRE].

[26] W. Fu, D. Gaiotto, J. Maldacena and S. Sachdev, Supersymmetric Sachdev-Ye-Kitaev models, Phys. Rev. D 95 (2017) 026009 [arXiv:1610.08917] [InSPIRE].

[27] E. Witten, An SYK-like model without disorder, arXiv:1610.09758 [INSPIRE].

[28] R. Gurau, The complete $1 / N$ expansion of a SYK-like tensor model, Nucl. Phys. B 916 (2017) 386 [arXiv: 1611.04032] [INSPIRE].

[29] J.S. Cotler et al., Black holes and random matrices, JHEP 05 (2017) 118 [Erratum ibid. 09 (2018) 002] [arXiv: 1611.04650] [INSPIRE].

[30] I.R. Klebanov and G. Tarnopolsky, Uncolored random tensors, melon diagrams and the Sachdev-Ye-Kitaev models, Phys. Rev. D 95 (2017) 046004 [arXiv:1611.08915] [InSPIRE].

[31] R.A. Davison et al., Thermoelectric transport in disordered metals without quasiparticles: The Sachdev-Ye-Kitaev models and holography, Phys. Rev. B 95 (2017) 155131 [arXiv: 1612.00849] [INSPIRE].

[32] C. Peng, M. Spradlin and A. Volovich, A supersymmetric SYK-like tensor model, JHEP 05 (2017) 062 [arXiv: 1612.03851] [INSPIRE].

[33] C. Krishnan, S. Sanyal and P.N. Bala Subramanian, Quantum chaos and holographic tensor models, JHEP 03 (2017) 056 [arXiv: 1612.06330] [INSPIRE].

[34] G. Turiaci and H. Verlinde, Towards a $2 d$ QFT analog of the SYK model, JHEP 10 (2017) 167 [arXiv: 1701.00528] [INSPIRE].

[35] T. Li, J. Liu, Y. Xin and Y. Zhou, Supersymmetric SYK model and random matrix theory, JHEP 06 (2017) 111 [arXiv:1702.01738] [INSPIRE]. 
[36] R. Gurau, Quenched equals annealed at leading order in the colored SYK model, EPL 119 (2017) 30003 [arXiv: 1702.04228] [INSPIRE].

[37] G. Mandal, P. Nayak and S.R. Wadia, Coadjoint orbit action of Virasoro group and two-dimensional quantum gravity dual to SYK/tensor models, JHEP 11 (2017) 046 [arXiv: 1702.04266] [INSPIRE].

[38] V. Bonzom, L. Lionni and A. Tanasa, Diagrammatics of a colored SYK model and of an SYK-like tensor model, leading and next-to-leading orders, J. Math. Phys. 58 (2017) 052301 [arXiv: 1702.06944] [INSPIRE].

[39] D.J. Gross and V. Rosenhaus, The bulk dual of SYK: cubic couplings, JHEP 05 (2017) 092 [arXiv: 1702.08016] [INSPIRE].

[40] D. Stanford and E. Witten, Fermionic localization of the Schwarzian theory, JHEP 10 (2017) 008 [arXiv: 1703.04612] [inSPIRE].

[41] C. Krishnan, K.V.P. Kumar and S. Sanyal, Random matrices and holographic tensor models, JHEP 06 (2017) 036 [arXiv:1703.08155] [INSPIRE].

[42] J. Maldacena, D. Stanford and Z. Yang, Diving into traversable wormholes, Fortsch. Phys. 65 (2017) 1700034 [arXiv: 1704.05333] [INSPIRE].

[43] S.R. Das, A. Jevicki and K. Suzuki, Three dimensional view of the SYK/AdS duality, JHEP 09 (2017) 017 [arXiv: 1704.07208] [INSPIRE].

[44] P. Narayan and J. Yoon, SYK-like tensor models on the lattice, JHEP 08 (2017) 083 [arXiv: 1705.01554] [INSPIRE].

[45] S. Chaudhuri et al., Abelian tensor models on the lattice, Phys. Rev. D 97 (2018) 086007 [arXiv: 1705.01930] [INSPIRE].

[46] T.G. Mertens, G.J. Turiaci and H.L. Verlinde, Solving the Schwarzian via the conformal bootstrap, JHEP 08 (2017) 136 [arXiv:1705.08408] [INSPIRE].

[47] J. Murugan, D. Stanford and E. Witten, More on supersymmetric and $2 d$ analogs of the SYK model, JHEP 08 (2017) 146 [arXiv:1706. 05362] [INSPIRE].

[48] C. Krishnan and K.V.P. Kumar, Towards a finite-N hologram, JHEP 10 (2017) 099 [arXiv: 1706. 05364] [INSPIRE].

[49] D.J. Gross and V. Rosenhaus, A line of CFTs: from generalized free fields to SYK, JHEP 07 (2017) 086 [arXiv: 1706.07015] [INSPIRE].

[50] A. Eberlein, V. Kasper, S. Sachdev and J. Steinberg, Quantum quench of the Sachdev-Ye-Kitaev model, Phys. Rev. B 96 (2017) 205123 [arXiv:1706.07803] [InSPIRE].

[51] M. Taylor, Generalized conformal structure, dilaton gravity and SYK, JHEP 01 (2018) 010 [arXiv: 1706. 07812] [INSPIRE].

[52] A.M. García-García, B. Loureiro, A. Romero-Bermúdez and M. Tezuka, Chaotic-integrable transition in the Sachdev-Ye-Kitaev model, Phys. Rev. Lett. 120 (2018) 241603 [arXiv: 1707. 02197] [INSPIRE].

[53] I. Kourkoulou and J. Maldacena, Pure states in the SYK model and nearly-AdS $S_{2}$ gravity, arXiv: 1707.02325 [INSPIRE].

[54] D. Anninos, T. Anous and R.T. D'Agnolo, Marginal deformations $\mathcal{E}$ rotating horizons, JHEP 12 (2017) 095 [arXiv:1707.03380] [INSPIRE]. 
[55] S. Giombi, I.R. Klebanov and G. Tarnopolsky, Bosonic tensor models at large $N$ and small

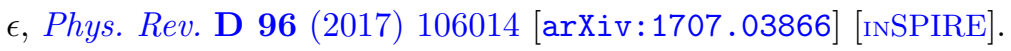

[56] J. Sonner and M. Vielma, Eigenstate thermalization in the Sachdev-Ye-Kitaev model, JHEP 11 (2017) 149 [arXiv: 1707.08013] [INSPIRE].

[57] K. Bulycheva, I.R. Klebanov, A. Milekhin and G. Tarnopolsky, Spectra of operators in large $N$ tensor models, Phys. Rev. D 97 (2018) 026016 [arXiv:1707.09347] [INSPIRE].

[58] S. Choudhury et al., Notes on melonic $O(N)^{q-1}$ tensor models, JHEP 06 (2018) 094 [arXiv: 1707.09352] [INSPIRE].

[59] D. Grumiller et al., Menagerie of AdS 2 boundary conditions, JHEP 10 (2017) 203 [arXiv: 1708.08471] [INSPIRE].

[60] D.J. Gross and V. Rosenhaus, All point correlation functions in SYK, JHEP 12 (2017) 148 [arXiv: 1710.08113] [INSPIRE].

[61] A. Kitaev and S.J. Suh, The soft mode in the Sachdev-Ye-Kitaev model and its gravity dual, JHEP 05 (2018) 183 [arXiv: 1711.08467] [INSPIRE].

[62] S.R. Das, A. Ghosh, A. Jevicki and K. Suzuki, Three dimensional view of arbitrary q SYK models, JHEP 02 (2018) 162 [arXiv:1711.09839] [INSPIRE].

[63] P. Narayan and J. Yoon, Supersymmetric SYK model with global symmetry, JHEP 08 (2018) 159 [arXiv: 1712. 02647] [inSPIRE].

[64] S.R. Das, A. Ghosh, A. Jevicki and K. Suzuki, Space-time in the SYK model, JHEP 07 (2018) 184 [arXiv: 1712.02725] [InSPIRE].

[65] H.A. González, D. Grumiller and J. Salzer, Towards a bulk description of higher spin SYK, JHEP 05 (2018) 083 [arXiv: 1802.01562] [INSPIRE].

[66] C. Krishnan and K.V. Pavan Kumar, Exact solution of a strongly coupled gauge theory in 0 1 dimensions, Phys. Rev. Lett. 120 (2018) 201603 [arXiv:1802.02502] [INSPIRE].

[67] D.A. Roberts, D. Stanford and A. Streicher, Operator growth in the SYK model, JHEP 06 (2018) 122 [arXiv: 1802.02633] [INSPIRE].

[68] D. Benedetti and R. Gurau, 2PI effective action for the SYK model and tensor field theories, JHEP 05 (2018) 156 [arXiv:1802.05500] [INSPIRE].

[69] A. Gaikwad, L.K. Joshi, G. Mandal and S.R. Wadia, Holographic dual to charged SYK from $3 D$ gravity and Chern-Simons, arXiv:1802.07746 [INSPIRE].

[70] I.R. Klebanov, A. Milekhin, F. Popov and G. Tarnopolsky, Spectra of eigenstates in fermionic tensor quantum mechanics, Phys. Rev. D 97 (2018) 106023 [arXiv:1802.10263] [INSPIRE].

[71] K.S. Kolekar and K. Narayan, $A d S_{2}$ dilaton gravity from reductions of some nonrelativistic theories, Phys. Rev. D 98 (2018) 046012 [arXiv: 1803.06827] [INSPIRE].

[72] H. Gharibyan, M. Hanada, S.H. Shenker and M. Tezuka, Onset of random matrix behavior in scrambling systems, JHEP 07 (2018) 124 [Erratum ibid. 02 (2019) 197] [arXiv: 1803.08050] [INSPIRE].

[73] J. Maldacena and X.-L. Qi, Eternal traversable wormhole, arXiv:1804.00491 [INSPIRE].

[74] D. Harlow and D. Jafferis, The factorization problem in Jackiw-Teitelboim gravity, arXiv:1804.01081 [INSPIRE]. 
[75] A.R. Brown et al., Falling toward charged black holes, Phys. Rev. D 98 (2018) 126016 [arXiv: 1804.04156] [INSPIRE].

[76] H.T. Lam, T.G. Mertens, G.J. Turiaci and H. Verlinde, Shockwave S-matrix from Schwarzian quantum mechanics, JHEP 11 (2018) 182 [arXiv:1804.09834] [INSPIRE].

[77] I. Bena, P. Heidmann and D. Turton, AdS $S_{2}$ holography: mind the cap, JHEP 12 (2018) 028 [arXiv: 1806.02834] [INSPIRE].

[78] P. Saad, S.H. Shenker and D. Stanford, A semiclassical ramp in SYK and in gravity, arXiv: 1806.06840 [INSPIRE].

[79] S.S. Gubser, C. Jepsen, Z. Ji and B. Trundy, Higher melonic theories, JHEP 09 (2018) 049 [arXiv: 1806.04800] [INSPIRE].

[80] F. Larsen, A nAttractor mechanism for $n A d S_{2} / n C F T_{1}$ holography, JHEP 04 (2019) 055 [arXiv: 1806. 06330] [INSPIRE].

[81] A. Blommaert, T.G. Mertens and H. Verschelde, The Schwarzian theory - A Wilson line perspective, JHEP 12 (2018) 022 [arXiv:1806.07765] [INSPIRE].

[82] C.-M. Chang, S. Colin-Ellerin and M. Rangamani, On melonic supertensor models, JHEP 10 (2018) 157 [arXiv: 1806. 09903] [INSPIRE].

[83] G. Gur-Ari, R. Mahajan and A. Vaezi, Does the SYK model have a spin glass phase?, JHEP 11 (2018) 070 [arXiv: 1806.10145] [INSPIRE].

[84] A. Goel, H.T. Lam, G.J. Turiaci and H. Verlinde, Expanding the black hole interior: partially entangled thermal states in SYK, JHEP 02 (2019) 156 [arXiv:1807.03916] [INSPIRE].

[85] J. Lin, Entanglement entropy in Jackiw-Teitelboim gravity, arXiv:1807.06575 [INSPIRE].

[86] A. Castro, F. Larsen and I. Papadimitriou, $5 D$ rotating black holes and the $n A d S_{2} / n C F T_{1}$ correspondence, JHEP 10 (2018) 042 [arXiv:1807.06988] [INSPIRE].

[87] J. Liu, E. Perlmutter, V. Rosenhaus and D. Simmons-Duffin, d-dimensional SYK, AdS Loops and 6j Symbols, JHEP 03 (2019) 052 [arXiv: 1808.00612] [INSPIRE].

[88] S. Giombi et al., Prismatic large $N$ models for bosonic tensors, Phys. Rev. D 98 (2018) 105005 [arXiv: 1808.04344] [INSPIRE].

[89] A. Kitaev and S.J. Suh, Statistical mechanics of a two-dimensional black hole, JHEP 05 (2019) 198 [arXiv: 1808.07032] [InSPIRE].

[90] K. Pakrouski, I.R. Klebanov, F. Popov and G. Tarnopolsky, Spectrum of Majorana quantum mechanics with $O(4)^{3}$ symmetry, Phys. Rev. Lett. 122 (2019) 011601 [arXiv: 1808.07455] [INSPIRE].

[91] M. Blake, R.A. Davison, S. Grozdanov and H. Liu, Many-body chaos and energy dynamics in holography, JHEP 10 (2018) 035 [arXiv:1809.01169] [INSPIRE].

[92] Z. Yang, The quantum gravity dynamics of near extremal black holes, JHEP 05 (2019) 205 [arXiv: 1809.08647] [INSPIRE].

[93] A.R. Brown et al., Complexity of Jackiw-Teitelboim gravity, Phys. Rev. D 99 (2019) 046016 [arXiv: 1810.08741] [INSPIRE].

[94] K.S. Kolekar and K. Narayan, On AdS 2 holography from redux, renormalization group flows and c-functions, JHEP 02 (2019) 039 [arXiv:1810.12528] [INSPIRE]. 
[95] F. Larsen and Y. Zeng, Black hole spectroscopy and AdS 2 holography, JHEP 04 (2019) 164 [arXiv: 1811.01288] [INSPIRE].

[96] R. Bhattacharya, D.P. Jatkar and N. Sorokhaibam, Quantum quenches and thermalization in SYK models, JHEP 07 (2019) 066 [arXiv: 1811.06006] [INSPIRE].

[97] M. Alishahiha, On complexity of Jackiw-Teitelboim gravity, Eur. Phys. J. C 79 (2019) 365 [arXiv: 1811.09028] [INSPIRE].

[98] A. Dhar et al., Gravitational collapse in SYK models and Choptuik-like phenomenon, arXiv: 1812.03979 [INSPIRE].

[99] J. Murugan and H. Nastase, One-dimensional bosonization and the SYK model, JHEP 08 (2019) 117 [arXiv: 1812.11929] [InSPIRE].

[100] K. Goto et al., Holographic complexity equals which action?, JHEP 02 (2019) 160 [arXiv: 1901.00014] [INSPIRE].

[101] J. Kim, I.R. Klebanov, G. Tarnopolsky and W. Zhao, Symmetry breaking in coupled SYK or tensor models, Phys. Rev. X 9 (2019) 021043 [arXiv: 1902.02287] [INSPIRE].

[102] S. Sachdev, Universal low temperature theory of charged black holes with AdS $S_{2}$ horizons, J. Math. Phys. 60 (2019) 052303 [arXiv: 1902 .04078] [inSPIRE].

[103] A. Blommaert, T.G. Mertens and H. Verschelde, Clocks and rods in Jackiw-Teitelboim quantum gravity, JHEP 09 (2019) 060 [arXiv:1902.11194] [INSPIRE].

[104] P. Nayak, J. Sonner and M. Vielma, Eigenstate thermalisation in the conformal Sachdev-Ye-Kitaev model: an analytic approach, JHEP 10 (2019) 019 [arXiv:1903.00478] [INSPIRE].

[105] F. Sun, Y. Yi-Xiang, J. Ye and W.M. Liu, Classification of the quantum chaos in colored Sachdev-Ye-Kitaev models, arXiv:1903.02213 [INSPIRE].

[106] V. Jahnke, K.-Y. Kim and J. Yoon, On the chaos bound in rotating black holes, JHEP 05 (2019) 037 [arXiv: 1903.09086] [InSPIRE].

[107] P. Saad, S.H. Shenker and D. Stanford, JT gravity as a matrix integral, arXiv:1903.11115 [INSPIRE].

[108] T.G. Mertens, Towards black hole evaporation in Jackiw-Teitelboim gravity, JHEP 07 (2019) 097 [arXiv: 1903.10485] [inSPIRE].

[109] J. Maldacena, G.J. Turiaci and Z. Yang, Two dimensional nearly de Sitter gravity, arXiv:1904.01911 [INSPIRE].

[110] H. Guo, Y. Gu and S. Sachdev, Transport and chaos in lattice Sachdev-Ye-Kitaev models, Phys. Rev. B 100 (2019) 045140 [arXiv: 1904.02174] [INSPIRE].

[111] T.G. Mertens and G.J. Turiaci, Defects in Jackiw-Teitelboim quantum gravity, JHEP 08 (2019) 127 [arXiv: 1904.05228] [inSPIRE].

[112] L. Susskind, Complexity and Newton's laws, arXiv:1904.12819 [INSPIRE].

[113] H.W. Lin, J. Maldacena and Y. Zhao, Symmetries near the horizon, JHEP 08 (2019) 049 [arXiv: 1904.12820] [INSPIRE].

[114] L.V. Iliesiu, S.S. Pufu, H. Verlinde and Y. Wang, An exact quantization of Jackiw-Teitelboim gravity, arXiv: 1905.02726 [INSPIRE]. 
[115] J. Cotler, K. Jensen and A. Maloney, Low-dimensional de Sitter quantum gravity, arXiv: 1905.03780 [INSPIRE].

[116] I.R. Klebanov, P.N. Pallegar and F.K. Popov, Majorana fermion quantum mechanics for higher rank tensors, Phys. Rev. D 100 (2019) 086003 [arXiv: 1905.06264] [INSPIRE].

[117] F. Sun and J. Ye, Periodic table of SYK and supersymmetric SYK, arXiv:1905.07694 [INSPIRE].

[118] V. Rosenhaus, An introduction to the SYK model, arXiv:1807.03334 [INSPIRE].

[119] G. Sárosi, $A d S_{2}$ holography and the $S Y K$ model, PoS (Modave2017) 001 [arXiv:1711.08482] [INSPIRE].

[120] M. Guica, T. Hartman, W. Song and A. Strominger, The Kerr/CFT Correspondence, Phys. Rev. D 80 (2009) 124008 [arXiv: 0809.4266] [InSPIRE].

[121] T. Hartman, K. Murata, T. Nishioka and A. Strominger, CFT duals for extreme black holes, JHEP 04 (2009) 019 [arXiv:0811.4393] [INSPIRE].

[122] T. Hartman, W. Song and A. Strominger, Holographic derivation of Kerr-Newman scattering amplitudes for general charge and spin, JHEP 03 (2010) 118 [arXiv:0908.3909] [INSPIRE].

[123] I. Bredberg, T. Hartman, W. Song and A. Strominger, Black hole superradiance from Kerr/CFT, JHEP 04 (2010) 019 [arXiv:0907.3477] [INSPIRE].

[124] M. Guica and A. Strominger, Microscopic realization of the Kerr/CFT correspondence, JHEP 02 (2011) 010 [arXiv: 1009.5039] [INSPIRE].

[125] A. Castro, A. Maloney and A. Strominger, Hidden conformal symmetry of the Kerr black hole, Phys. Rev. D 82 (2010) 024008 [arXiv: 1004.0996] [InSPIRE].

[126] A.P. Porfyriadis and A. Strominger, Gravity waves from the Kerr/CFT correspondence, Phys. Rev. D 90 (2014) 044038 [arXiv:1401.3746] [INSPIRE].

[127] S. Hadar, A.P. Porfyriadis and A. Strominger, Gravity waves from extreme-mass-ratio plunges into Kerr black holes, Phys. Rev. D 90 (2014) 064045 [arXiv:1403.2797] [INSPIRE].

[128] S. Hadar, A.P. Porfyriadis and A. Strominger, Fast plunges into Kerr black holes, JHEP 07 (2015) 078 [arXiv: 1504.07650] [inSPIRE].

[129] A.P. Porfyriadis, Y. Shi and A. Strominger, Photon emission near extreme kerr black holes, Phys. Rev. D 95 (2017) 064009 [arXiv:1607.06028] [INSPIRE].

[130] G. Compère, The Kerr/CFT correspondence and its extensions, Living Rev. Rel. 15 (2012) 11 [arXiv: 1203.3561] [INSPIRE].

[131] S.W. Hawking and S.F. Ross, Duality between electric and magnetic black holes, Phys. Rev. D 52 (1995) 5865 [hep-th/9504019] [INSPIRE].

[132] J.M. Maldacena, J. Michelson and A. Strominger, Anti-de Sitter fragmentation, JHEP 02 (1999) 011 [hep-th/9812073] [INSPIRE].

[133] A. Sen, Black hole entropy function and the attractor mechanism in higher derivative gravity, JHEP 09 (2005) 038 [hep-th/0506177] [INSPIRE].

[134] A. Sen, Black hole entropy function, attractors and precision counting of microstates, Gen. Rel. Grav. 40 (2008) 2249 [arXiv:0708.1270] [INSPIRE]. 
[135] K. Goldstein, N. Iizuka, R.P. Jena and S.P. Trivedi, Non-supersymmetric attractors, Phys. Rev. D 72 (2005) 124021 [hep-th/0507096] [INSPIRE].

[136] R.P. Kerr, Gravitational field of a spinning mass as an example of algebraically special metrics, Phys. Rev. Lett. 11 (1963) 237 [INSPIRE].

[137] J.M. Bardeen and G.T. Horowitz, The extreme Kerr throat geometry: a vacuum analog of $A d S_{2} \times S^{2}$, Phys. Rev. D 60 (1999) 104030 [hep-th/9905099] [INSPIRE].

[138] R.H. Boyer and R.W. Lindquist, Maximal analytic extension of the Kerr metric, J. Math. Phys. 8 (1967) 265 [INSPIRE].

[139] D. Astefanesei et al., Rotating attractors, JHEP 10 (2006) 058 [hep-th/0606244] [INSPIRE].

[140] A. Castro and F. Larsen, Near extremal kerr entropy from AdS $S_{2}$ quantum gravity, JHEP 12 (2009) 037 [arXiv: 0908.1121] [inSPIRE].

[141] S.W. Hawking, C.J. Hunter and M.M. Taylor-Robinson, Rotation and the AdS/CFT correspondence, Phys. Rev. D 59 (1999) 064005 [hep-th/9811056] [INSPIRE].

[142] G.W. Gibbons, H. Lü, D.N. Page and C.N. Pope, The general Kerr-de Sitter metrics in all dimensions, J. Geom. Phys. 53 (2005) 49 [hep-th/0404008] [INSPIRE].

[143] G.W. Gibbons, H. Lü, D.N. Page and C.N. Pope, Rotating black holes in higher dimensions with a cosmological constant, Phys. Rev. Lett. 93 (2004) 171102 [hep-th/0409155] [INSPIRE].

[144] S.W. Hawking and H.S. Reall, Charged and rotating AdS black holes and their CFT duals, Phys. Rev. D 61 (2000) 024014 [hep-th/9908109] [INSPIRE].

[145] H.K. Kunduri, J. Lucietti and H.S. Reall, Gravitational perturbations of higher dimensional rotating black holes: Tensor perturbations, Phys. Rev. D 74 (2006) 084021 [hep-th/0606076] [INSPIRE].

[146] B. Carter, Hamilton-Jacobi and Schrödinger separable solutions of Einstein's equations, Commun. Math. Phys. 10 (1968) 280 [INSPIRE].

[147] J.F. Plebański and M. Demiański, Rotating, charged and uniformly accelerating mass in general relativity, Annals Phys. 98 (1976) 98 [INSPIRE].

[148] V. Cardoso, O.J.C. Dias, J.P.S. Lemos and S. Yoshida, The Black hole bomb and superradiant instabilities, Phys. Rev. D 70 (2004) 044039 [Erratum ibid. D 70 (2004) 049903] [hep-th/0404096] [INSPIRE].

[149] V. Cardoso and O.J.C. Dias, Small Kerr-anti-de Sitter black holes are unstable, Phys. Rev. D 70 (2004) 084011 [hep-th/0405006] [INSPIRE].

[150] V. Cardoso, O.J.C. Dias and S. Yoshida, Classical instability of Kerr-AdS black holes and the issue of final state, Phys. Rev. D 74 (2006) 044008 [hep-th/0607162] [INSPIRE].

[151] M.M. Caldarelli, G. Cognola and D. Klemm, Thermodynamics of Kerr-Newman-AdS black holes and conformal field theories, Class. Quant. Grav. 17 (2000) 399 [hep-th/9908022] [INSPIRE].

[152] V.A. Kostelecký and M.J. Perry, Solitonic black holes in gauged $N=2$ supergravity, Phys. Lett. B 371 (1996) 191 [hep-th/9512222] [INSPIRE].

[153] M.M. Caldarelli and D. Klemm, Supersymmetry of Anti-de Sitter black holes, Nucl. Phys. B 545 (1999) 434 [hep-th/9808097] [INSPIRE]. 
[154] J.E. McClintock et al., The spin of the near-extreme kerr black hole GRS 1915+105, Astrophys. J. 652 (2006) 518 [astro-ph/0606076] [INSPIRE].

[155] J.L. Blum et al., Measuring the spin of GRS 1915+105 with relativistic disk reflection, Astrophys. J. 706 (2009) 60 [arXiv:0909.5383] [INSPIRE].

[156] J.M. Miller et al., NuSTAR spectroscopy of GRS 1915+105: disk reflection, spin and connections to jets, Astrophys. J. 775 (2013) L45 [arXiv: 1308.4669] [INSPIRE].

[157] S. Bhattacharyya, V.E. Hubeny, S. Minwalla and M. Rangamani, Nonlinear Fluid Dynamics from Gravity, JHEP 02 (2008) 045 [arXiv: 0712.2456] [INSPIRE].

[158] U. Moitra, S.P. Trivedi and V. Vishal, unpublished (2018).

[159] G.W. Gibbons, M.J. Perry and C.N. Pope, The first law of thermodynamics for Kerr-anti-de Sitter black holes, Class. Quant. Grav. 22 (2005) 1503 [hep-th/0408217] [INSPIRE]. 